\title{
On Bogoliubov Transformations. II. The General Case*
}

\author{
S. N. M. RuIJSENAARS \\ Department of Physics, Princeton University, Princeton, New Jersey 08540
}

Received January 19, 1978

\begin{abstract}
A rigorous treatment of Bogoliubov transformations is presented along the same lines as in a previous paper, which dealt with a special case. As in the previous paper a formulation in terms of unitary resp. pseudo-unitary operators is used, corresponding to the CAR resp. the CCR. This leads to simple proofs of well-known necessary and sufficient conditions for the transformation to be unitarily implementable in Fock space. The normal form of the implementing operator $\mathscr{U}$ is studied. It is proved that on the subspace of algebraic tensors $\mathscr{U}$ equals a strongly convergent infinite series of Wick monomials that sums up to a simple exponential expression. A connection between the fermion and boson transformations studied in the previous paper is established. The analogous correspondence in the general case only holds true if the (pseudo) unitary operator equals its own inverse.
\end{abstract}

\section{INTRODUCTION}

In a previous paper [1] we studied the special type of Bogoliubov transformation that occurs in the treatment of systems of relativistic, charged particles. It is the purpose of the present paper to consider general Bogoliubov transformations in a similar fashion. In particular, as in [1], our main objective is to study the normal form of the unitary operator on Fock space that implements the transformation.

In Section 2 we collect some results on second quantization we have occasion to use. The two definitions of Bogoliubov transformation that can be found in the literature are discussed and their relation is established. In Section 3 we present our own approach in terms of unitary resp. pseudo-unitary operators that commute with the "charge conjugation operator," corresponding to the canonical anticommutation relations (CAR) resp. canonical commutation relations (CCR). This approach and our notation are inspired by the way Bogoliubov transformations occur in theories of relativistic, neutral particles in external fields [2]. At first sight it may seem somewhat artificial to readers who are only familiar with applications of Bogoliubov transformations to nonrelativistic theories. However, it will be seen that it leads to an easy proof of a well-known necessary condition for the transformation to be unitarily implement-

\footnotetext{
* Work supported in part by NSF Contract MPS 74-22844.
} 
able (Theorem 3.1) and to simple expressions for the implementing operators (Theorems 5.3 and 6.3). By using this formalism we also show that the transformation should be invertible if it is to be unitarily implementable. The approach leads to a formulation of the Weyl and Clifford algebras that differs slightly from the usual one (cf. (3.5)-(3.13)).

In Section 4 we introduce an operator $A$ which is closely related to the (pseudo) unitary operator $U$ that generates the transformation. It is in terms of this operator (which we call the associate of $U$ ) that the implementing operator $\mathscr{U}$ on Fock space can be easily expressed. We derive several properties of the associate and the pure creation part of $\mathscr{U}$. These are used in Section 5 to prove that the transformation is unitarily implementable if $U$ satisfies the assumptions made below. We then proceed to obtain a simple expression for the normal form of $\mathscr{Z}$. That such an expression exists is plausible from earlier work on Bogoliubov transformations (cf. the book by Berezin [3]). However, in general a bounded operator on Fock space only admits a normal form in a quite weak distributional sense. In the present case we prove that $\mathscr{U}$ admits a normal form in the strong sense of it being equal on the subspace $D$ of algebraic tensors to a strongly convergent infinite series of Wick monomials. More in particular, we show that the a priori formal expression

$$
\left.: \exp \left(\frac{1}{2}\left[\Lambda_{+-} c^{*} c^{*}+\Lambda_{++} c^{*} c+\Lambda_{--} c c^{*}+\Lambda_{-+} c c\right]\right): \quad \text { (cf. }(5.14)\right)
$$

has a rigorous mathematical meaning as an operator mapping $D$ into the subspace of $C^{\infty}$-vectors for the number operator, and that this operator is equal to $\mathbb{U}$ divided by its vacuum expectation value. In the fermion case there is a well-known restriction on $U$, which is dropped in Section 6. Here it is proved that then $\mathscr{U}$ still exists, and its somewhat more involved normal form is obtained.

The last section, Section 7 , contains the derivation of the main results of [1] from the general results obtaincd in this paper. Moreover, a remarkable connection between the boson and fermion transformations studied in [1] is pointed out. In the general case the natural analog of this correspondence only holds true if the operator $U$ equals its own inverse in addition to other more general restrictions.

As a result of our approach the methods and proofs to be presented below are rather similar to those that can be found in [1]. However, instead of referring to [1] we have usually preferred to give complete proofs, as they are actually more transparent in the general case considered here. We have also presented a short proof of the wellknown fact that $\mathscr{U}$ exists if the transformed annihilation operators have a vacuum since the existing proofs known to us are in our opinion either rather difficult and indirect, or not fully satisfactory as regards the domain difficulties involved here (that is, in the boson case). In the same spirit we have included a proof of the irreducibility of the Weyl operators $\exp (i R(f))$, requiring nothing more than Stone's theorem and a characterization of the domain of the Segal field operators $R(f)$.

The functional analysis background required for this paper can be found in the books by Reed and Simon $[4,5]$. References to earlier work on Bogoliubov transformations are given in the main text. 


\section{Preliminaries}

Although our results could be stated and proved in a coordinate free way, it turns out to be very convenient to employ a realization of Fock space as a space of sequences of square-integrable functions. Accordingly, we assume that the Hilbert space $\mathscr{H}_{+}$on which Fock space is built is given by

$$
\mathscr{H}_{+}=L^{2}\left(R^{m}, d p\right)^{M}, \quad 1 \leqslant m, M<\infty,
$$

i.e., it consists of $M$-component square-integrable functions on $R^{m}$. The coordinatefree analogs of the results can be obtained in the same fashion as sketched in [1]. It is easy to see that they also hold when $\mathscr{H}_{+}$has finite dimension.

The (anti-) symmetric Fock space $\mathscr{F}_{\epsilon}$ over $\mathscr{H}_{+}(\epsilon=a, s)$ consists of sequences of square-integrable (anti-) symmetric functions $\left\{\phi^{n}\left(p_{1}, \alpha_{1}, \ldots, p_{n}, \alpha_{n}\right)\right\}_{n=0}^{\infty}$, satisfying

$$
(\phi, \phi)=\sum_{n=0}^{\infty} \sum_{\alpha_{1}, \ldots, \alpha_{n}=1}^{M} \int d p_{1} \cdots d p_{n}\left|\phi^{n}\left(p_{1}, \alpha_{1}, \ldots, p_{n}, \alpha_{n}\right)\right|^{2}<\infty .
$$

The subspace of vectors $\phi$ for which only a finite number of $\phi^{n}$ is nonzero will be denoted by $D_{f}$. The creation and annihilation operators $c^{*}(f)$ and $c(f)$, depending linearly resp. antilinearly on vectors $f \in \mathscr{H}_{+}$, are defined on $D_{f}$ by

$$
\begin{aligned}
& (c(f) \phi)^{n}\left(p_{1}, \alpha_{1}, \ldots, p_{n}, \alpha_{n}\right) \\
& \quad=(n+1)^{1 / 2} \sum_{\alpha=1}^{M} \int d p \bar{f}_{\alpha}(p) \phi^{n+1}\left(p, \alpha, p_{1}, \alpha_{1}, \ldots, p_{n}, \alpha_{n}\right), \\
& \left(c^{*}(f) \phi\right)^{n}\left(p_{1}, \alpha_{1}, \ldots, p_{n}, \alpha_{n}\right) \\
& \quad=n^{-1 / 2} \sum_{i=1}^{n}(\mp)^{i+1} f_{\alpha_{i}}\left(p_{i}\right) \phi^{n-1}\left(p_{1}, \alpha_{1}, \ldots, \hat{p}_{i}, \hat{\alpha}_{i}, \ldots, p_{n}, \alpha_{n}\right) .
\end{aligned}
$$

The indices will be suppressed from now on. It easily follows from (2.3) that on $D_{f}$ the CAR (CCR) hold:

$$
\begin{aligned}
{[c(f), c(g)]_{ \pm} } & =\left[c^{*}(f), c^{*}(g)\right]_{ \pm}=0, \\
{\left[c(f), c^{*}(g)\right]_{ \pm} } & =(f, g), \quad \forall f, g \in \mathscr{H}_{+} .
\end{aligned}
$$

In (2.3) and (2.4), as in the whole paper, the upper (lower) sign refers to the fermion (boson) case. From (2.3) one concludes

$$
c^{*}(f) \phi=c(f)^{*} \phi, \quad \forall \phi \subset D_{f} .
$$

Using this and (2.4) it follows that the $c^{(*)}(f)$ are bounded in the fermion case. It is not difficult to deduce from (2.3) that the $c^{(*)}(f)$ are unbounded in the boson case, but in view of (2.5) they are closable. In both cases we denote the closures by the same symbols. Note that $(2.5)$ and the CCR imply that $D(c(f))=D\left(c^{*}(f)\right)$. 
On $D_{f}$ we obviously have

$$
\begin{aligned}
c(f) P_{M+1} & =P_{M} c(f), \\
c^{*}(f) P_{M-1} & =P_{M} c^{*}(f),
\end{aligned}
$$

where $P_{M}$ is the projection of the number operator $N \equiv d \Gamma(1)$ on $[0, M]$. Hence, these relations also hold on $D(c(f))$, and

$$
c^{(*)}(f) \phi=s \cdot \lim _{M \rightarrow \infty} c^{(*)}(f) P_{M} \phi, \quad \forall \phi \in D(c(f))=D\left(c^{*}(f)\right) .
$$

Therefore (2.3) holds for any vector in $D(c(f))$. We further note that for any $\phi \in D$ $\left(c(f)^{*}\right)$ and $\psi \in D_{f}$ one has $\left(P_{M} c(f)^{*} \phi, \psi\right)=\left(\phi, c(f) P_{M} \psi\right)=\left(c^{*}(f) P_{M-1} \phi, \psi\right)$, so that $P_{M} c(f)^{*} \phi=c^{*}(f) P_{M-1} \phi$. Thus

$$
c^{*}(f)=c(f)^{*} .
$$

We now introduce the Segal field operators

$$
R(f)=2^{-1 / 2}\left[c(f)+c^{*}(f)\right] .
$$

Obviously, $R(f)$ is self-adjoint in the fermion case. In the boson case one concludes from Nelson's analytic vector theorem that $R(f)$ is essentially self-adjoint on $D_{f}$ (see Reed and Simon [5], p. 210). We denote the closure by the same symbol. Setting

$$
L_{f} \equiv\left\{\phi \mid s \cdot \lim _{M \rightarrow \infty}\left[c(f) P_{M+1}+c^{*}(f) P_{M-1}\right] \phi \text { exists }\right\}
$$

we claim that

$$
\begin{gathered}
L_{f}=D(R(f)), \\
R(f) \phi=s \cdot \lim _{M \rightarrow \infty} 2^{-1 / 2}\left[c(f) P_{M+1}+c^{*}(f) P_{M-1}\right] \phi, \quad \forall \phi \in D(R(f)) .
\end{gathered}
$$

Indeed, in view of (2.6) we have on $D_{f}$ and hence on $D(R(f))$

$$
P_{M} R(f)=2^{-1 / 2}\left[c(f) P_{M+1}+c^{*}(f) P_{M-1}\right] .
$$

It follows from this that $L_{f} \supset D(R(f))$ and that (2.12) holds. On the other hand, if $\phi \in L_{f}$, then for any $\psi \in D_{f}$ one has

$$
\begin{aligned}
\left(\phi^{*}, \psi\right) & \equiv \lim _{M} 2^{-1 / 2}\left(\left[c(f) P_{M+1}+c^{*}(f) P_{M-1}\right] \phi, \psi\right) \\
& =\lim _{M} 2^{-1 / 2}\left(\phi,\left[c(f)+c^{*}(f)\right] P_{M} \psi\right)=(\phi, R(f) \psi),
\end{aligned}
$$

and so $\phi \in D(R(f))$. Therefore (2.11) holds as well. From (2.12) and (2.7) it follows that

$$
D(R(f)) \cap D(R(i f))=D(c(f)),
$$

so that we have

$$
c(f)=2^{-1 / 2}[R(f)+i R(i f)]
$$


The Segal field operators satisfy the commutation relations

$$
\begin{aligned}
& {[R(f), R(g)]_{+}=\operatorname{Re}(g)} \\
& {[R(f), R(g)]_{-}=i \operatorname{Im}(f, g) \quad \text { (bosons). }}
\end{aligned}
$$

Relation (2.17) holds on $D_{f}$. It implies (see [5], 1.c.)

$$
\exp [i R(f)] \exp [i R(g)]=\exp [i \operatorname{Im}(g, f)] \exp [i R(g)] \exp [i R(f)] \text {. }
$$

The $R(f)$ (fermions) resp. $\exp [i R(f)]$ (bosons) are irreducible: if a bounded operator $A$ commutes with all of them, it must be a multiple of the identity. Indeed, if (fermions) $A R(f)=R(f) A, \forall f \in \mathscr{H}_{+}$, then from (2.15) $A c^{(*)}(f)=c^{(*)}(f) A, \forall f \in \mathscr{H}_{+}$, and so $c(f) A \Omega=A c(f) \Omega=0, \forall f \in \mathscr{H}_{+}$, where $\Omega$ is the vacuum. Thus, in view of $(2.3)$ one must have $A \Omega=\alpha \Omega, \alpha \in C$. But then $A \prod_{i=1}^{n} c^{*}\left(f_{i}\right) \Omega=\prod_{i=1}^{n} c^{*}\left(f_{i}\right) A \Omega=\alpha$ $\prod_{i=1}^{n} c^{*}\left(f_{i}\right) \Omega$, so that $A=\alpha$. If (bosons) $A \exp [i R(f)]=\exp [i R(f)] A, \forall f \in \mathscr{H}_{+}$, then because of Stone's theorem $A D(R(f)) \subset D(R(f))$ and $A R(f)=R(f) A, \forall f \in \mathscr{H}_{+}$. Thus, by (2.14), $A D(c(f)) \subset D(c(f))$ and $A c^{(*)}(f)=c^{(*)}(f) A, \forall f \in \mathscr{H}_{+}$. Hence, by the same argument as for fermions, $A=\alpha$.

Besides $D_{f}$ we need three other dense subspaces of $\mathscr{F}_{\epsilon}$ : the intersection of the domains of all creation and annihilation operators, or equivalently (cf. (2.14)) of all Segal field operators,

$$
\tilde{D} \equiv \bigcap_{f \in \mathscr{A}_{+}} D(c(f))=\bigcap_{f \in \mathscr{\varkappa}_{+}} D(R(f))
$$

the subspace of $C^{\infty}$-vectors for $N$,

$$
D_{\infty}=\bigcap_{k=1}^{\infty} D\left(N^{k}\right)
$$

and the subspace of vectors that arc finite linear combinations of vectors of the form $\prod_{i=1}^{n} c^{*}\left(f_{i}\right) \Omega$, denoted by $D$. By using (2.6)-(2.7) and the easily verified relations

$$
\begin{aligned}
c(f) c^{*}(f) P_{M} & \leqslant\|f\|^{2}(N+1) P_{M}, \\
c(f) N^{k} P_{M} & =(N+1)^{k} c(f) P_{M},
\end{aligned}
$$

one infers that $D^{\infty} \subset \tilde{D}$ and that $D^{\infty}$ is left invariant by the $c^{(*)}(f)$. From (2.6) it then follows that

$$
\begin{aligned}
\prod_{i=1}^{n} c^{(*)}\left(f_{i}\right) \phi & =\lim _{M} P_{M} \prod_{i=1}^{n} c^{(*)}\left(f_{i}\right) \phi=\lim _{M} c^{(*)}\left(f_{1}\right) P_{M} \prod_{i=2}^{n} c^{(*)}\left(f_{i}\right) \phi=\cdots \\
& =\lim _{M} \prod_{i=1}^{n} c^{(*)}\left(f_{i}\right) P_{M} \phi, \quad \forall \phi \in D_{\infty} .
\end{aligned}
$$

As a result, the CCR hold on $D_{\infty}$.

In the literature Bogoliubov transformations are introduced in two different ways, roughly speaking. The first version gocs back to Bogoliubov [6]. In this form the 
transformation has been applied by many authors to solid-state physics, statistical mechanics, and relativistic quantum field theory, in particular the external field problem. Purely mathematical studies using this formalism can be found in the books by Friedrichs [7] and Berezin [3], and in [8]. One introduces new annihilation and creation operators

$$
\begin{aligned}
\hat{c}(f) & \equiv c\left(T_{1} f\right)+c^{*}\left(\overline{T_{2} f}\right), \\
\hat{c}^{*}(f) & \equiv c^{*}\left(T_{1} f\right)+c\left(\overline{T_{2} f}\right),
\end{aligned}
$$

where $T_{1}$ and $T_{2}$ are complex-linear operators on $\mathscr{H}_{1}$, such that the $\hat{c}^{(*)}$ again satisfy the CAR (CCR), i.e., such that

$$
\begin{aligned}
& T_{1}^{*} \bar{T}_{2} \pm T_{2}^{*} \bar{T}_{1}=0, \\
& T_{1}^{*} T_{1} \pm T_{2}^{*} T_{2}=1 .
\end{aligned}
$$

In (2.24) the bar denotes complex conjugation, and for any operator $A$ on $\mathscr{H}_{+}, \bar{A}$ is defined by

$$
\bar{A} f=\overline{A \bar{f}} .
$$

The second approach, introduced by Segal (cf. [9] and references therein), has also been used by many authors. Here, as a rule, the transformations are considered in a wider algebraic context. Since we only deal with the Fock representation of the relevant algebras, we refrain from describing this algebraic framework (cf. e.g., [10] and references given there). In the more special Fock space context one considers transformations of the Segal field operators

$$
\hat{R}(f) \equiv R(T f),
$$

where $T$ is a real-linear operator on $\mathscr{H}_{+}$such that (2.16) resp. (2.17) (or equivalently (2.18)) also hold for the $\hat{R}$. Thus, in the fermion case $T$ is required to be an orthogonal operator, i.e., an operator preserving the real inner product $\operatorname{Re}(f, g)$, while in the boson case $T$ must be a symplectic operator, i.e., an operator preserving the symplectic form $\operatorname{Im}(f, g)$.

Setting

$$
\begin{aligned}
T f & =\frac{1}{2}(T-i T i) f+\frac{1}{2}(T+i T i) f \\
& \equiv T_{1} f+\overline{T_{2} f},
\end{aligned}
$$

one easily verifies that $T_{1}$ and $T_{2}$ are complex-linear, and that the transformation of the $c^{(*)}$, induced by setting

$$
\hat{R}(f) \equiv 2^{-1 / 2}\left[\hat{c}(f)+\hat{c}^{*}(f)\right]
$$

amounts to (2.24), and vice versa. Moreover, a straightforward calculation shows that $T$ is orthogonal/symplectic (in the sense defined above) if and only if the operators $T_{1}$ and $T_{2}$, defined by (2.28), satisfy $(2.25)$. 
In the first formulation the transformation is by definition unitarily implementable if a unitary operator $\mathscr{U}$ exists, satisfying

$$
\hat{c}^{(*)}(f)=\mathscr{U}^{*} c^{(*)}(f) \mathscr{U}, \quad \forall f \in \mathscr{H}_{+} .
$$

In the boson case we require that this hold at least on $D_{f}$, so that $\mathscr{U}$ maps $D_{f}$ into $\tilde{D}$. In the second formulation one requires instead that (fermions)

$$
\hat{R}(f)=\mathscr{U}^{*} R(f) \mathscr{U}, \quad \forall f \in \mathscr{H}_{+},
$$

which is clearly equivalent to (2.30), resp. (bosons)

$$
\exp [i \hat{R}(f)]=\mathscr{U}^{*} \exp [i R(f)] \mathscr{U}, \quad \forall f \in \mathscr{H}_{+} .
$$

If (2.30) holds on $D_{f}$, then (2.31) holds on $D_{f}$ as well, and so (2.32) holds by virtue of the functional calculus. Conversely, if (2.32) holds then by Stone's theorem $\mathscr{U}$ maps $D_{f}$ into $\tilde{D}$ and (2.31) holds on $D_{f}$. Therefore (2.30) holds on $D_{f}$.

We conclude that the two approaches are fully equivalent (that is, for our purposes). We note further that if $\mathscr{U}$ exists it is uniquely determined up to a phase factor because of the irreducibility of the $R(f)$ resp. $\exp [i R(f)]$.

In the above we have implicitly assumed that the operators $T_{1}, T_{2}$, and $T$ are everywhere defined and closed, and as such bounded. Starting with $a$ priori unbounded closed operators, defined on a dense subspace, one readily concludes from (2.25) that $T_{1}$ and $T_{2}$ must be bounded in the fermion case. However, in the boson case one can easily construct an example of two unbounded operators satisfying (2.25) on a dense subspace. In a similar fashion as in [1], it can be seen that under some natural assumptions concerning domains such transformations cannot be unitarily implementable. Since we are only interested in implementable transformations, we assume from now on that we are dealing with bounded operators.

In the literature it is usually part of the definition that the transformation is invertible, i.e., that one can write the $c^{(*)}$ in terms of the $\hat{c}^{(*)}$, or equivalently that $T$ is invertible. We shall presently show that this is necessarily true if the transformation is unitarily implementable.

\section{Formulation in Terms of Unitary and Pseudo-Unitary Operators}

In this section we present a third formulation of Bogoliubov transformations. Instead of the real-linear Segal field operators (2.9) we propose to consider other field operators that are complex-linear. It is in terms of the latter field operators that Bogoliubov transformations naturally arise in theories of neutral, relativistic particles in external fields [2]. More importantly, this approach immediately leads to a description in terms of the familiar class of complex-linear unitary resp. pseudo-unitary operators, as opposed to the less intuitive class of real-linear orthogonal resp. symplectic operators. It will be seen that this leads to a quite simple proof of Theorem 3.1 (which has a rather more difficult proof in the latter version) and to simple expressions 
for the implementing operators. It will also enable us to easily compare our results with the results obtained for the special type of Bogoliubov transformation that occurs in theories of relativistic, charged particles [1].

To this end we introduce a Hilbert space $\mathscr{H}$, which is the direct sum of two subspaces $\mathscr{H}_{+}$and $\mathscr{H}_{-}$, with corresponding projections $P_{+}$and $P_{-} . \mathscr{H}_{+}$is the space that we have employed thus far, and $\mathscr{H}_{-}$is a copy of it, i.e., it also satisfies (2.1). Thus, a vector $v \in \mathscr{H}$ is given by two ( $M$-component) square-integrable functions $v_{+}(p)$ and $v_{-}(p)$. We define a conjugation $C$ on $\mathscr{H}$ by

$$
(C v)_{\epsilon}(p)=\bar{v}_{-\epsilon}(p), \quad \epsilon=+,-
$$

and a unitary, self-adjoint operator $q$ by

It is convenient to set

$$
q=P_{+}-P_{-} .
$$

$$
A_{\mathrm{\epsilon \varepsilon}^{\prime}}=P_{\epsilon^{\prime}} A P_{\epsilon^{\prime}}
$$

if $A$ is a bounded operator on $\mathscr{H}$. Note that

$$
A_{\mathrm{\epsilon \epsilon}}{ }^{*}=A_{\epsilon^{\prime} \epsilon}^{*} .
$$

We now introduce field operators on $\tilde{D}$ by

$$
\psi(v)=c\left(P_{+} v\right)+c^{*}\left(C_{-} v\right), \quad \forall v \in \mathscr{H} .
$$

Note that the r.h.s. is well defined since $C P_{-} v \in \mathscr{H}_{+}$. Clearly, one has on $\tilde{D}$

$$
\psi(v)^{*}=\psi(C v) .
$$

The field operators satisfy the further algebraic relations

$$
\begin{array}{ll}
{\left[\psi(u), \psi(v)^{*}\right]_{+}=(u, v),} & \text { (fermions), } \\
{\left[\psi(u), \psi(v)^{*}\right]_{-}=(u, q v),} & \text { (bosons), }
\end{array}
$$

where (3.8) holds on $D_{\infty}$. We shall denote the closures of the $\psi$ by the same symbol. Note that $\psi(v)$ equals $c\left(P_{+} v\right)$ resp. $c^{*}\left(C_{-} v\right)$ if $P_{-} v$ resp. $P_{+} v$ is zero. Moreover, setting

one concludes that

$$
\mathscr{H}_{c} \equiv\{v \in \mathscr{H} \mid C v=v\}
$$

Conversely,

$$
\psi(v)=2^{1 / 2} R\left(P_{+} v\right), \quad \forall v \in \mathscr{H}_{\mathrm{c}}
$$

$$
R(f)=2^{-1 / 2} \psi(f+C f), \quad \forall f \in \mathscr{H}_{+} .
$$

Thus, the $\psi(v)$ resp. $\exp [i \psi(v)]$, where $v \in \mathscr{H}_{c}$, are irreducible and we have

$$
\tilde{D}=\bigcap_{v \in \mathscr{H}_{p}} D(\psi(v))
$$


As the analog of (2.18) one obtains

$$
\begin{array}{r}
\exp [i \psi(u)] \exp [i \psi(v)]=\exp [(v, q u)] \exp [i \psi(v)] \exp [i \psi(u)], \\
\forall u, v \in \mathscr{H}_{c} .
\end{array}
$$

Note further that in the relation

$$
\psi(v)=\frac{1}{2} \psi(v \cdot+C v)+\frac{1}{2} i \psi(i[v-C v]), \quad \forall v \in \mathscr{H},
$$

both arguments on the r.h.s. belong to $\mathscr{H}_{c}$.

Let us consider transformations of the field operators,

$$
\hat{\psi}(v)=\psi\left(U^{*} v\right), \quad \forall v \in \mathscr{H},
$$

generated by bounded complex-linear operators $U$ on $\mathscr{H}$. For which operators do the $\hat{\psi}(v)$ again satisfy (3.6) and (3.7) resp. (3.8)? We immediately obtain as necessary and sufficient conditions for this that

and that

$$
C U=U C
$$

$$
\begin{aligned}
U U^{*} & =1, & & \text { (fermions), } \\
U q U^{*} & =q, & & \text { (bosons). }
\end{aligned}
$$

We now ask: for which $U$ is there a unitary operator $\mathscr{U}$ such that

$$
\hat{\psi}(v)=\mathscr{U}^{*} \psi(v) \mathscr{U}, \quad \forall v \in \mathscr{H} .
$$

In the boson case we require that this relation hold on $D_{f}$. If such an operator exists, we will say that the transformation is unitarily implementable. It is obvious that this can only be the case if (3.16) and (3.17) hold. (In the boson case we can equivalently require

$$
\exp [i \hat{\psi}(v)]=\mathscr{U}^{*} \exp [i \psi(v)] \mathscr{U}, \quad \forall v \in \mathscr{H}_{c} .
$$

Indeed, if (3.18) holds, then $U^{*}$ maps $\mathscr{H}_{c}$ into $\mathscr{H}_{c}$ since (3.16) must hold. By using the functional calculus, (3.19) follows. Conversely, if (3.19) holds, then $U^{*}$ maps $\mathscr{H}_{c}$ into $\mathscr{H}_{e}$, and so it commutes with $C$. Therefore (3.14) also holds for $\hat{\psi}$, so that (3.18) follows from Stone's theorem.) We claim that a further necessary condition for (3.18) to hold is that $U$ satisfy

$$
\begin{aligned}
U^{*} U & =1, & & \text { (fermions), } \\
U^{*} q U & =q, & & \text { (bosons). }
\end{aligned}
$$

Indeed, if $A$ and $B$ are bounded operators satisfying $A B=1$ and $\operatorname{Ran} B$ is dense it easily follows that $B A=1$. Thus, since (3.17) must hold, it suffices to prove that $M \equiv \operatorname{Ran} U^{*}$ is dense in $\mathscr{H}$. Since (3.16) must hold as well, we have $C P_{+} M-P_{-} M$. Hence, $M$ is dense in $\mathscr{H}$ if and only if $P_{+} M$ is dense in $\mathscr{H}_{+}$. Let $f \in \mathscr{H}_{+}$be orthogonal to $M$, then we are through if we prove that $f$ must be zero. Since also $C f$ is orthogonal 
to $M$ one has for any $v \in \mathscr{H}_{c}$ (fermions) $\psi(v) \mathscr{U} c^{*}(f) c(f) \mathscr{U}^{*}=\mathscr{U} \psi\left(U^{*} v\right) \psi(C f) \psi$ $(f) \mathscr{U}^{*}=\mathscr{U} c^{*}(f) c(f) \mathscr{U}^{*} \psi(v)$ resp. (bosons) $\exp [i \psi(v)] \mathscr{U} \exp [i \psi(f+C f)] \mathscr{U}^{*}=$ $\mathscr{U} \exp \left[i \psi\left(U^{*} v\right)\right] \exp [i \psi(f+C f)] \mathscr{U}^{*}=\mathscr{U} \exp [i \psi(f+C f)] \mathscr{U}^{*} \exp [i \psi(v)]$, where (3.6) and (3.7) resp. (3.13) have been used. Using irreducibility it follows that $c^{*}(f) c(f)$ resp. $\exp [i \psi(f+C f)]$ are constants, so that $f$ must be zero.

As noted above, (3.19) a priori only implies that $\mathscr{U}$ maps $D_{f}$ into $\tilde{D}$ if it exists. However, we have just shown that it implies (3.20). As a result $U^{*}$ maps $\mathscr{H}_{c}$ onto $\mathscr{H}_{c}$. Therefore $\mathscr{U}$ actually maps $\tilde{D}$ onto $\tilde{D}$ if it exists.

The three conditions we have obtained are not yet sufficient for $\mathscr{U}$ to exist. However, before proving a further necessary condition we shall show that the present approach is equivalent to the first approach mentioned in Section 2 (and so to the second one as well). Let us first assume that (2.24) and (2.25) hold. We can then define an operator $U$ on $\mathscr{H}$ by setting

$$
\begin{array}{ll}
U_{++} \equiv T_{1}^{*}, & U_{+-} \equiv T_{2}^{*}, \\
U_{--} \equiv \overline{U_{++}}, & U_{-+} \equiv \overline{U_{+-}} .
\end{array}
$$

Here we have identified $\mathscr{H}_{-}$with $\mathscr{H}_{+}$in the obvious way (we shall often do this in the sequel). It is an immediate consequence of (3.22) that (3.16) holds, and (2.25) implies that (3.17) holds.

On the other hand, if $U$ satisfies (3.16) and (3.17), and we define $T_{1}$ and $T_{2}$ by (3.21), then (2.25) follows. Hence, there is a one-one correspondence between Bogoliubov transformations and field operator transformations generated by $U$ satisfying (3.16) and (3.17). Moreover, under this correspondence (3.18) holds if and only if (2.30) holds. Indeed, if (3.21)-(3.22) hold it is straightforward to verify that

$$
\hat{\psi}(v)=\hat{c}\left(P_{+} v\right)+\hat{c}^{*}\left(C_{-} \_v\right), \quad \forall v \in \mathscr{H},
$$

from which our assertion evidently follows. Summarizing we conclude that the two approaches are fully equivalent.

So far, we have shown that if (3.18) is to hold we must have

and

$$
C U=U C
$$

$$
\begin{aligned}
U U^{*} & =U^{*} U=1, & & \text { (fermions), } \\
U q U^{*} & =U^{*} q U=q, & & \text { (bosons), }
\end{aligned}
$$

i.e., $U$ has to be a unitary resp. pseudo-unitary (w.r.t. $q$ ) operator on $\mathscr{H}$, commuting with $C$. We can also write (3.25) as

$$
\begin{aligned}
& U^{*}{ }_{++} U_{++} \pm U^{*}{ }_{+-} U_{-+}=U_{++} U^{*}{ }_{++} \pm U_{+-} U^{*}{ }_{-+}=1_{++} \text {, } \\
& U^{*}{ }_{--} U_{--} \pm U_{{ }_{+}}^{*} U_{+-}=U_{--} U^{*}{ }_{--} \pm U_{-+} U_{+-}^{*}=1_{--} \text {, } \\
& U^{*}{ }_{++} U_{+-} \pm U^{*}{ }_{+-} U_{--}=U_{++} U^{*}{ }_{+-} \pm U_{+-} U^{*}=0 \text {, } \\
& U^{*}{ }_{--} U_{-+} \pm U^{*}{ }_{-+} U_{++}=U_{--} U^{*}{ }_{-+} \pm U_{-+} U^{*}{ }_{++}=0 \text {. }
\end{aligned}
$$


From now on we assume that (3.24) and (3.25) (or equivalently (3.26)) are satisfied.

It is convenient to consider also the transformation generated by the inverse of $U$ :

$$
\begin{aligned}
& \psi^{\prime}(v)=\psi(U v), \quad \text { (fermions), } \\
& \psi^{\prime}(v)=\psi(q U q v), \quad \text { (bosons). }
\end{aligned}
$$

The corresponding transformed annihilation and creation operators are then given by

$$
\begin{aligned}
c^{\prime}(f) & =c\left(U_{++} f\right) \pm c^{*}\left(C U_{-+} f\right), \\
c^{\prime *}(f) & =c^{*}\left(U_{++} f\right) \pm c\left(C U_{-+} f\right) .
\end{aligned}
$$

If $\mathscr{U}$ is a unitary operator, mapping $\tilde{D}$ onto $\tilde{D}$, which satisfies (3.18) on $\tilde{D}$, then it is clear that $\mathscr{U}$ also satisfies

and

$$
\psi^{\prime}(v)=\mathscr{U} \psi(v) \mathscr{U}^{*}, \quad \forall v \in \mathscr{H}
$$

$$
c^{\prime}(f)=\mathscr{U} c(f) \mathscr{U}^{*}, \quad \forall f \in \mathscr{H}_{+}
$$

on $\tilde{D}$, and vice versa. Now (3.30) implies $c^{\prime}(f) \mathscr{U} \Omega=\mathscr{U} c(f) \Omega=0, \forall f \in \mathscr{H}_{+}$. Thus, it is necessary for (3.30) that a nonzero vector $\Omega^{\prime} \in \tilde{D}$ exist, satisfying $c^{\prime}(f) \Omega^{\prime}=0$, $\forall f \in \mathscr{H}_{+}$. We shall now prove that such a vector can only exist if $U_{+-}$is HilbertSchmidt (H.S.). It follows from Theorem 5.2 and 6.2 that this is also sufficient for $\mathscr{U}$ to exist. This H.S. condition is well known. It dates back to semirigorous work of Friedrichs in the early fifties [7]. The first rigorous proof of an equivalent condition in the boson case is due to Shale [11], in the fermion case to Shale and Stinespring [12]. Both proofs were based on earlier results of Segal. Thereafter several authors proved similar conditions in different contexts. Unfortunately, the existing necessity proofs are, in our opinion, either rather complicated or partly formal. The following proof is both simple and rigorous.

THEOREM 3.1. Let $U$ be a bounded operator on $\mathscr{H}$, satisfying (3.24) and (3.25). Assume a nonzero vector $\Omega^{\prime} \in \tilde{D}$ exists, satisfying

where

$$
c^{\prime}(f) \Omega^{\prime}=0, \quad \forall f \in \mathscr{H}_{+}
$$

$$
c^{\prime}(f) \equiv c\left(U_{++} f\right) \pm c^{*}\left(C U_{-+} f\right) .
$$

Then $U_{-+}$is Hilbert-Schmidt.

Proof. A. Bosons. ${ }^{1}$ We define projections $P^{n}(n \in Z)$ by (cf. (2.2))

$$
\left(P^{n} \phi\right)^{n^{\prime}}=\delta_{n n^{\prime}} \phi^{n} \text {. }
$$

${ }^{1}$ After completion of the preprint of this paper B. Simon informed the author that the proof given below for the boson case already appeared in his unpublished 1972 lecture notes on Bogoliubov transformations. He learned the proof from A. S. Wightman. 
Since $P^{n}=P_{n}-P_{n-1}$, it follows from (2.6) that

$$
\begin{aligned}
c\left(U_{++} f\right) P^{n+2} \Omega^{\prime} & =P^{n+1} c\left(U_{++} f\right) \Omega^{\prime}=P^{n+1} c^{*}\left(C U_{-+} f\right) \Omega^{\prime} \\
& =c^{*}\left(C U_{-+} f\right) P^{n} \Omega^{\prime} .
\end{aligned}
$$

It is an easy consequence of the first line of (3.26) that $\operatorname{Ran} U_{++}$equals $\mathscr{H}_{+}$. Therefore, if $P^{n_{0}} \Omega^{\prime}=0$ with $n_{0} \neq-2$, then according to (3.34) $P^{n_{0}+2} \Omega^{\prime}=0$. Hence,

$$
P^{n} \Omega^{\prime}=0, \quad n \text { odd. }
$$

But then, since $\Omega^{\prime}$ is nonzero:

$$
P^{0} \Omega^{\prime} \equiv \alpha \neq 0 \text {. }
$$

Setting $\psi(p, q) \equiv \alpha^{-1}\left(P^{2} \Omega^{\prime}\right)^{2}(p, q)$ and using (3.34) and (2.3) we infer

$$
\left(C U_{-+} f\right)(q)=2^{1 / 2} \int d p\left(\overline{U_{++} f}\right)(p) \psi(p, q) .
$$

Since $\psi$ is square-integrable, $U_{-+}$is H.S. in view of (3.37).

B. Fermions. If Ran $U_{++}$would be dense in $\mathscr{H}_{+}$, the proof would be the same as for bosons. However, it is easy to see that this is not true in general. This circumstance makes the proof somewhat more difficult, but the basic idea is the same. It is convenient to use the following notation: if $A$ is a bounded operator on $\mathscr{H}$ we denote by $\operatorname{Ker} A_{\varepsilon \varepsilon}$ the kernel of $A_{\varepsilon \varepsilon}$, viewed as an operator on $\mathscr{H}_{\epsilon}$. Note that then

$$
\operatorname{Ker} A_{\epsilon \epsilon} \oplus \overline{\operatorname{Ran} A_{\epsilon \epsilon}^{*}}=\mathscr{H}_{\epsilon} .
$$

Let $\left\{f_{i}\right\}_{i=1}^{L}$ and $\left\{f_{i}\right\}_{i=L+1}^{\infty}$ be orthonormal (o.n.) bases for Ker $U_{++}^{*}$ resp. $\overline{\operatorname{Ran}} \overline{U_{++}}$. Then $\left\{f_{i}\right\}_{i=1}^{\infty}$ is an o.n. base for $\mathscr{H}_{+}$and so the vectors

$$
\phi_{p_{1}, \rho_{2}, \ldots} \equiv \prod_{i=1}^{\infty} c^{*}\left(f_{i}\right)^{\rho_{i}} \Omega
$$

where

$$
\rho_{i}=0,1, \quad \sum_{i=1}^{\infty} \rho_{i}<\infty
$$

are an o.n. base for $\mathscr{F}_{a}\left(\mathscr{H}_{+}\right)$. Hence,

$$
\Omega^{\prime}=-\sum_{\rho_{i}} \alpha_{p_{1}, \rho_{2}, \ldots} \phi_{o_{1}, \rho_{2}, \ldots} .
$$

Now if $f \in \operatorname{Ker} U_{++}^{*}$, then $g \equiv U^{*} f=U^{*}{ }_{-+} f$ belongs to $\mathscr{H}_{-}$, and according to (3.26), $U_{-} g=U_{--} U_{-+}^{*} f=-U_{-+} U_{++}^{*} f=0$. Thus, $f=U g=U_{+-}$. Hence, using (3.24),

$$
c^{\prime}(C g)=c\left(U_{++} C g\right)+c^{*}\left(C U_{-+} C g\right)=c\left(C U_{--} g\right)+c^{*}\left(U_{+-} g\right)=c^{*}(f) .
$$


From (3.31) it now follows that

$$
c^{*}\left(f_{i}\right) \Omega^{\prime}=0, \quad i=1, \ldots, L .
$$

This clearly implies that $L<\infty$, and that

$$
\sum_{i=1}^{L} \rho_{i}<L \rightarrow \alpha_{\rho_{1}, \rho_{2}, \ldots}=0 .
$$

As a result,

$$
P^{n} \Omega^{\prime}=0, \quad \forall n<L
$$

and

$$
P^{L} \Omega^{\prime}=\beta \prod_{i=1}^{L} c^{*}\left(f_{i}\right) \Omega
$$

The analog of (3.34) is

$$
c\left(U_{++} f\right) P^{n+2} \Omega^{\prime}=-c^{*}\left(C U_{-+} f\right) P^{n} \Omega^{\prime} .
$$

Reasoning as in A, and using (3.44) we infer that

$$
P^{L+n} \Omega^{\prime}=0, \quad n \text { odd }
$$

and that $\beta \neq 0$ in (3.46). Now according to (3.44) we have

$$
P^{L+2} \Omega^{\prime}=\frac{1}{2} \sum_{k, l=L+1}^{\infty} \gamma_{k l} c^{*}\left(f_{k}\right) c^{*}\left(f_{l}\right) P^{L} \Omega^{\prime}
$$

where

$$
\gamma_{k l}=-\gamma_{l k}, \quad \sum_{k, l=L+1}^{\infty}\left|\gamma_{k l}\right|^{2}<\infty .
$$

By using (3.47) and the CAR it follows that

$$
\sum_{k, l} \gamma_{k l}\left(U_{++} f, f_{k}\right) c^{*}\left(f_{l}\right) P^{L} \Omega^{\prime}=-c^{*}\left(C U_{-+} f\right) P^{L} \Omega^{\prime} .
$$

If $f \in \operatorname{Ran} U_{++}^{*}$, then $C U_{-+} f=C U_{-+} U_{++}^{*} g=-C U_{--} U_{++}^{*} g=-U_{++} U_{+-}^{*} C g$, so $C U_{-+} f \in \operatorname{Ran} U_{++}$. In view of (3.46) and (3.51) we thus have

$$
\sum_{k, l} \gamma_{k l}\left(U_{++} f, f_{k}\right) c^{*}\left(f_{l}\right) \Omega=-c^{*}\left(C U_{-+} f\right) \Omega, \quad \forall f \in \operatorname{Ran} U_{++}^{*} .
$$

By continuity,

$$
C U_{-+} f=-\sum_{k, l} \gamma_{k l} f_{l}\left(U_{++} f, f_{k}\right), \quad \forall f \in \overline{\operatorname{Ran} U_{++}^{*}}
$$


However, by using (3.24) and (3.26) one easily verifies that

$$
\operatorname{Ker} U_{++}=C U^{*} \operatorname{Ker} U_{++}^{*}
$$

and therefore $\operatorname{Ker} U_{++}$has dimension $L<\infty$. Combining this with (3.53) and (3.50) we conclude that $U_{-+}$is H.S.

From now on we assume in addition to (3.24) and (3.25) that $U_{-+}$is H.S. Because of (3.24) $U_{-+}$is then also H.S.

\section{The Operators $\Lambda$ AND $\exp \left(\frac{1}{2} \Lambda_{+} c^{*} c^{*}\right)$}

In the boson case $U_{++}$and $U_{--}$have bounded inverses (as operators on $\mathscr{H}_{+}$resp. $\mathscr{H}_{-}$). This easily follows from (3.26) by using their polar decomposition. In the fermion case this is also true, provided

$$
\operatorname{Ker} U_{++}=0
$$

(cf. the remark above (3.38)). This readily follows from (3.24), (3.26), and our assumption that $U_{-1}$ is H.S. In this section and the next we assume that (4.1) holds. In Section 6 we study the case $\operatorname{Ker} U_{++} \neq 0$.

It turns out that the implementing operator can be easily expressed in terms of a bounded operator $A$, defined by

$$
\begin{array}{ll}
\Lambda_{--}= \pm\left(1_{--}-U_{--}^{-1}\right), & \Lambda_{+-}=U_{+-} U_{-_{-}^{-1}}^{-1} \\
\Lambda_{-+}= \pm U_{--}^{-1} U_{-+}, & \Lambda_{++}=U_{++}-1_{++}-U_{+-} U_{--}^{-1} U_{-+} .
\end{array}
$$

We will call $\Lambda$ the associate of $U$. In view of (3.26) we can equivalently define $A$ by

$$
\begin{array}{ll}
\Lambda_{--}= \pm\left(1_{--}-U_{--}^{*}+U^{*+} U_{++}^{*-1} U_{+-}^{*}\right), & \Lambda_{+-}=\mp U_{++}^{*-1} U_{+-}^{*}, \\
\Lambda_{-+}=-U_{-+}^{*} U_{++}^{*-1}, & \Lambda_{++}=-1_{++}+U_{++}^{*-1} .
\end{array}
$$

Finally, we can also define the associate of $U$ as the (uniquely determined) operator satisfying

$$
\begin{array}{ll}
\text { (fermions) } & U-1-\Lambda-(U-1) P_{-} \Lambda=U-1-\Lambda-\Lambda P_{-}(U-1)=0, \\
\text { (bosons) } & U-1-q \Lambda+(U-1) P_{-} \Lambda=U-1-q \Lambda-q \Lambda P_{-}(U-1)=0 .
\end{array}
$$

Because of (3.24), (4.2), and (4.3) we have

$$
\begin{aligned}
& \Lambda_{++}=\mp \Lambda_{--}{ }^{T}, \\
& \Lambda_{+-}=\mp \Lambda_{+-}{ }^{T}, \\
& \Lambda_{-+}=\mp \Lambda_{-+}{ }^{T} .
\end{aligned}
$$


In (4.6) all operators are regarded as operators on $\mathscr{H}_{+}$(cf. the remark below (3.22)). We will often do this in the sequel without further comment. The transpose of a bounded operator $A$ on $\mathscr{H}_{+}$is defined by

$$
A^{T}=\overline{A^{*}}
$$

We have occasion to use the relations

$$
\begin{gathered}
1_{++}+\Lambda_{++}=U_{++}-\Lambda_{+-} U_{-+}, \\
\Lambda_{-+} \mp U_{-+}+\Lambda_{--} U_{-+}=0
\end{gathered}
$$

which are immediate consequences of (4.2). It is also clear from (4.2) that $A_{+-}$and $\Lambda_{-+}$are H.S., and an easy argument, using (3.26), shows that

$$
\left\|\Lambda_{+-}\right\| \equiv \theta<1, \quad \text { (bosons). }
$$

We now claim that there exist o.n. sets $\left\{g_{n}\right\}$ resp. $\left\{g_{n}, h_{n}\right\}$ in $\mathscr{H}_{+}$such that

$$
\text { (bosons) } \quad A_{+-}=\sum_{n=1}^{M} \lambda_{n} g_{n} \otimes g_{n}, \quad 1>\theta=\lambda_{1} \geqslant \lambda_{2} \geqslant \cdots>0,
$$

$$
\text { (fermions) } \quad \Lambda_{+-}=\sum_{n=1}^{M} \lambda_{n}\left(g_{n} \otimes h_{n}-h_{n} \otimes g_{n}\right), \quad \lambda_{1} \geqslant \lambda_{2} \geqslant \cdots>0,
$$

where $M \leqslant \infty$ and

$$
\sum_{n=1}^{M} \lambda_{n}^{2}<\infty
$$

In (4.10) the symbol $f_{1} \otimes f_{2}\left(f_{1}, f_{2} \in \mathscr{H}_{+}\right)$denotes the operator

$$
\left(f_{1} \otimes f_{2}\right) f \equiv\left(\bar{f}_{2}, f\right) f_{1}, \quad \forall f \in \mathscr{H}_{+} .
$$

This assertion is a consequence of the following lemma. In the boson case this lemma is due to Kristensen, Mejlbo, and Thue Poulsen [8].

LeMma 4.1. Let $K$ be a compact operator on $\mathscr{H}_{+}$, satisfying

$$
K=K^{T}
$$

resp.

$$
K=-K^{T}
$$

Then there exist o.n. sets $\left\{g_{n}\right\}$ resp. $\left\{g_{n}, h_{n}\right\}$ and $M$ numbers $\lambda_{1} \geqslant \lambda_{2} \geqslant \cdots>0$ $(M \leqslant \infty)$ such that

$$
K=\sum_{n=1}^{M} \lambda_{n} g_{n} \otimes g_{n}
$$


resp.

$$
K=\sum_{n=1}^{M} \lambda_{n}\left(g_{n} \otimes h_{n}-h_{n} \otimes g_{n}\right)
$$

Proof. Since $K$ is compact there are $L$ numbers $\alpha_{1}>\alpha_{2}>\cdots>0(L \leqslant \infty)$ and $L$ partial isometries $U_{l}$ with mutually orthogonal finite-dimensional initial and final subspaces $\mathscr{H}_{i, l}$ resp. $\mathscr{H}_{f, l}$ (with corresponding projections $P_{l}$ resp. $Q_{l}$ ) such that

$$
K=\sum_{l=1}^{L} \alpha_{l} U_{l}
$$

In both cases the self-adjoint operators $K^{*} K$ and $\left(K K^{*}\right)^{T}$ are equal, and thus we must have

$$
P_{l}=Q_{l}^{T}, \quad l=1, \ldots, L .
$$

It easily follows from this that the $U_{l}$ satisfy (4.13) resp. (4.14). Therefore it suffices to prove the corresponding statements for a partial isometry $U$ with initial subspace $\mathscr{H}_{i}$ $\left(\operatorname{dim} \mathscr{H}_{i} \equiv n<\infty\right)$ and final subspace $\mathscr{H}_{f}$.

First, assume that $U=U^{T}$. Let $\phi$ be a nonzero vector in $\mathscr{H}_{f}$, then at least one of the vectors $\chi_{+} \equiv \phi+U \bar{\phi}, \chi_{-} \equiv i(\phi-U \bar{\phi})$ is nonzero. Since $U \bar{\chi}_{\epsilon}=\chi_{\epsilon}, \epsilon=+,-$, there exists a normalized vector $g$ such that $U \bar{g}=g$. Setting $U^{\prime}=U-g \otimes g$, it easily follows that $U^{\prime}$ is a partial isometry satisfying $U^{\prime}=U^{\prime T}$, but whose initial subspace has dimension $n-1$. The statement now follows by induction.

We now assume $U=-U^{T}$. Let $h$ be a normalized vector in $\mathscr{H}_{f}$ and set $g \equiv U \hbar$. Then one has $U \bar{g}=-h$ and $(g, h)=(U \bar{h}, h)=-(\hbar, \bar{U} h)=-(g, h)$, and so $g$ and $h$ are o.n. Hence $n \geqslant 2$, and setting $U^{\prime} \equiv U-(g \otimes h-h \otimes g)$ we conclude that $U^{\prime}$ is a partial isometry satisfying $U^{\prime}=-U^{\prime}$, but with initial subspace of dimension $n-2$. Again, induction completes the proof.

This lemma has the following easy corollary, which is of independent interest.

COROLlaRY 4.2. Let $H$ be an arbitrary Hilbert space. Let $\phi$ belong to $H \otimes^{8} H$ resp. $H \otimes^{a} H$. Then there exist o.n. sets $\left\{g_{n}\right\}$ resp. $\left\{g_{n}, h_{n}^{\prime}\right\}$ and $M$ numbers $\lambda_{1} \geqslant$ $\lambda_{2} \geqslant \cdots>0$ with $M \leqslant \infty$ and $\sum_{n=1}^{M} \lambda_{n}{ }^{2}<\infty$, such that

$$
\phi=\sum_{n=1}^{M} \lambda_{n} g_{n} \otimes g_{n}
$$

resp.

$$
\phi=\sum_{n=1}^{M} \lambda_{n}\left(g_{n} \otimes h_{n}-h_{n} \otimes g_{n}\right) .
$$

Since $\Lambda_{+-}$is H.S. the operator

$$
\Lambda_{+-} c^{*} c^{*} \equiv \int d p d q \Lambda_{+-}(p, q) c^{*}(p) c^{*}(q)
$$


(where $\Lambda_{+-}(p, q)$ is the kernel of $\Lambda_{+-}$) and its powers map $D_{f}$ into $D_{f}$. The next lemma shows that the expression $\exp \left(\frac{1}{2} \Lambda_{+-} c^{*} c^{*}\right)$ naturally defines an operator mapping $D$ into $D_{\infty}$.

LeMmA 4.3. Let $\phi \in D$, and let

$$
\phi_{n}=\frac{\left(\Lambda_{+-} c^{*} c^{*}\right)^{n}}{2^{n} n !} \phi
$$

Then $s \cdot \lim _{N \rightarrow \infty} \sum_{n=0}^{N} \phi_{n}$ exists and belongs to $D_{\infty}$.

Proof. A. Bosons. We first prove the lemma for $\phi=\Omega$. Clearly, the existence of the limit will then follow if we prove that the sequence

$$
a_{n}=\left(2^{n} n !\right)^{-2}\left\|\left(\Lambda_{+-} c^{*} c^{*}\right)^{n} \Omega\right\|^{2}
$$

is summable. To show this, we set

$$
a_{n, N}=\left(2^{n} n !\right)^{-2}\left\|\left(\Lambda_{N} c^{*} c^{*}\right)^{n} \Omega\right\|^{2},
$$

where

$$
\Lambda_{N} \equiv \sum_{i=\mathbf{1}}^{N} \lambda_{i} g_{i} \otimes g_{i}
$$

It is easy to see that

$$
\lim _{N \rightarrow \infty} a_{n, N}=a_{n}
$$

and that

$$
\begin{aligned}
a_{n, N} & -\left(2^{n} n !\right)^{-2}\left\|\left[\sum_{i=1}^{N} \lambda_{i} c^{*}\left(g_{i}\right)^{2}\right]^{n} \Omega\right\|^{2} \\
& =2^{-2 n}\left\|\sum_{\substack{k_{1} \ldots \ldots k_{N}=0 \\
k_{1}+\ldots+k_{N}=n}}^{n} \prod_{i=1}^{N}\left(k_{i} !\right)^{-1}\left[\lambda_{i} c^{*}\left(g_{i}\right)^{2}\right]^{k_{i}} \Omega\right\|^{2} \\
& =2^{-2 n} \sum_{\substack{k_{1}, \ldots, k_{N}=0 \\
k_{1} \ldots \ldots+k_{N}=n}}^{n} \prod_{i=1}^{N}\left(k_{i} !\right)^{-2}\left(2 k_{i}\right) ! \lambda_{i}^{2 k_{i}}
\end{aligned}
$$

We now introduce a function

$$
F_{N}(\alpha)=\prod_{i=1}^{N}\left(1-\alpha \lambda_{i}{ }^{2}\right)^{-1 / 2} .
$$

Because of (4.10), $F_{N}$ is analytic in the disc

$$
Q=\left\{\alpha \subset C \| \alpha \mid<\theta^{-2}\right\} .
$$


By comparing the relation

$$
F_{N}^{(n)}(0)=n ! \sum_{\substack{k_{1}, \ldots, k_{N}-0 \\ k_{1}+\ldots+k_{N}=n}}^{n} \prod_{\substack{j=1 \\ \text { nat }}}^{N}\left(k_{i} !\right)^{-1} 2^{-k_{i}}\left(2 k_{i}-1\right) ! ! \lambda_{i}^{2 k_{i}}
$$

with (4.27) it follows that

$$
F_{N}(\alpha)=\sum_{n=0}^{\infty} a_{n, N} \alpha^{n}
$$

By using the estimates (3.23)-(3.26) in [1] (with obvious changes) one now infers that

$$
\lim _{N \rightarrow \infty} F_{N}(\alpha)=F(\alpha), \quad \forall \alpha \in Q,
$$

where $F$ is analytic in $Q$ and where the convergence is uniform on compact subsets of $Q$. In view of (4.26) and (4.31) this implies that

$$
F(\alpha)=\sum_{n=0}^{\infty} a_{n^{\alpha^{n}}}
$$

and thus, since $\theta^{-2}>1$,

$$
\sum_{n=0}^{\infty} a_{n}<\infty
$$

Obviously,

$$
F(1)=\prod_{i=1}^{M}\left(1-\lambda_{i}^{2}\right)^{-1 / 2}
$$

and therefore (cf. [13])

$$
\text { (bosons) } \quad\left\|\exp \left(\frac{1}{2} \Lambda_{+-} c^{*} c^{*}\right) \Omega\right\|^{2}=\operatorname{det}\left(1-\Lambda_{+-}^{*} \Lambda_{+-}\right)^{-1 / 2} .
$$

Now the functions

$$
G_{k}(\alpha)=\sum_{n=0}^{\infty}(2 n)^{k} a_{n} \alpha^{n}, \quad k \in N^{+}
$$

are analytic in $Q$ since the power series on the r.h.s. have the same convergence radius as the r.h.s. of (4.33). Hence,

$$
\sum_{n=0}^{\infty}(2 n)^{k} a_{n}<\infty, \quad \forall k \in N^{+}
$$

from which we conclude

$$
\exp \left(\frac{1}{2} \Lambda_{+} c^{*} c^{*}\right) \Omega \in D_{\infty} .
$$

Thus, the lemma holds true if $\phi=\Omega$. 
We now observe that

$$
\sum_{n=0}^{N}\left(2^{n} n !\right)^{-1}\left(\Lambda_{+-} c^{*} c^{*}\right)^{n} \prod_{i=1}^{k} c^{*}\left(f_{i}\right) \Omega=\prod_{i=1}^{k} c^{*}\left(f_{i}\right) P_{2 N} \exp \left(\frac{1}{2} \Lambda_{+--} c^{*} c^{*}\right) \Omega
$$

From (4.39) and (2.23) it follows that the limit $N \rightarrow \infty$ of the r.h.s. of (4.40) exists and belongs to $D_{m}$. Hence the same is true for the 1.h.s. As a result the lemma holds true for any $\phi \in D$.

B. Fermions. Proceeding as in A we define as the analog of (4.25)

$$
\Lambda_{N}=\sum_{i=1}^{N} \lambda_{i}\left(g_{i} \otimes h_{i}-h_{i} \otimes g_{i}\right)
$$

As the analog of (4.27) we then obtain

$$
\begin{aligned}
a_{n, N} & =(n !)^{-2}\left\|\left[\sum_{i=1}^{N} \lambda_{i} c^{*}\left(g_{i}\right) c^{*}\left(h_{i}\right)\right]^{n} \Omega\right\|^{2} \\
& =\sum_{\substack{k_{1}, \ldots, k_{N}=0 \\
k_{1}+\ldots+k_{N}=n}}^{1} \prod_{i=1}^{N}\left[\lambda_{i} c^{*}\left(g_{i}\right) c^{*}\left(h_{i}\right)\right]^{k_{i}} \Omega \|^{2} \\
& =\sum_{\substack{k_{1}, \ldots . k_{N}=0 \\
k_{1}+\ldots+k_{N}=n}}^{1} \prod_{i=1}^{N} \lambda_{i}^{2 k_{i}} .
\end{aligned}
$$

Setting

$$
F_{N}(\alpha)=\prod_{i=1}^{N}\left(1+\alpha \lambda_{i}^{2}\right)
$$

it easily follows that (4.31) holds and by using the mentioned estimates from [1] we conclude that

$$
\lim _{N \rightarrow \infty} F_{N}(\alpha)=F(\alpha), \quad \forall \alpha \in C
$$

where $F$ is entire, and where the convergence is uniform on compact subsets of $C$. Thus, (4.34) holds and we have in place of (4.36)

$$
\text { (fermions) } \quad\left\|\exp \left(\frac{1}{2} \Lambda_{+-} c^{*} c^{*}\right) \Omega\right\|^{2}=\operatorname{det}\left(1+\Lambda_{+-}{ }^{*} \Lambda_{+-}\right)^{1 / 2} .
$$

The lemma now follows as in A. Of course, in this case existence of the limit of the r.h.s. of (4.40) immediately follows from the boundedness of the $c^{*}$.

CoRollary 4.4. For any $\phi \in D$ and $f \in \mathscr{H}_{+}$

$$
\begin{aligned}
& \exp \left(\frac{1}{2} \Lambda_{+-} c^{*} c^{*}\right) c^{*}(f) \phi=c^{*}(f) \exp \left(\frac{1}{2} \Lambda_{+-} c^{*} c^{*}\right) \phi \\
& \quad \exp \left(\frac{1}{2} \Lambda_{+-} c^{*} c^{*}\right) c(f) \phi-\left[c(f) \pm c^{*}\left(\Lambda_{+-} \bar{f}\right)\right] \exp \left(\frac{1}{2} \Lambda_{+-} c^{*} c^{*}\right) \phi
\end{aligned}
$$


Proof. It is sufficient to show this for a vector of the form $\phi=\prod_{i=1}^{n} c^{*}\left(f_{i}\right) \Omega$. By using the CAR (CCR) one then easily sees that

$$
\begin{aligned}
& P_{2 N+n+1} \exp (\cdots) c^{*}(f) \phi=c^{*}(f) P_{2 N+n} \exp (\cdots) \phi \\
& P_{2 N+n-1} \exp (\cdots) c(f) \phi=\left[c(f) P_{2 N+n} \pm c^{*}\left(\Lambda_{+-} \bar{f}\right) P_{2 N+n-2}\right] \exp (\cdots) \phi .
\end{aligned}
$$

(4.46) and (4.47) follow from this by taking $\lim N \rightarrow \infty$.

\section{The EXISTENCE of $\mathscr{U}$ AND Its Normal Form}

We begin this section with a proof that the $c^{\prime}(f)$ have a vacuum (cf. also $[7,3,8$, $14,15])$.

LEMMA 5.1. For any $f \in \mathscr{H}_{+}$one has

$$
c^{\prime}(f) \exp \left(\frac{1}{2} \Lambda_{+-} c^{*} c^{*}\right) \Omega=0 .
$$

Proof. We note first that the 1.h.s. is well defined in view of (4.39). Because of (4.47) and (4.2) we have

$$
\begin{aligned}
c\left(U_{++} f\right) \exp \left(\frac{1}{2} \Lambda_{+-} c^{*} c^{*}\right) \Omega & =\mp c^{*}\left(\Lambda_{+-} \overline{U_{++} f}\right) \exp \cdots \Omega \\
& =\mp c^{*}\left(\Lambda_{+-} U_{--} C f\right) \exp \cdots \Omega \\
& =\mp c^{*}\left(U_{+-} C f\right) \exp \cdots \Omega \\
& =\mp c^{*}\left(C U_{-+} f\right) \exp \left(\frac{1}{2} \Lambda_{+-} c^{*} c^{*}\right) \Omega .
\end{aligned}
$$

Therefore (5.1) holds.

We shall now show that the lemma implies that the transformation is unitarily implementable. We define

$$
\begin{gathered}
\mathscr{U} \Omega=\operatorname{det}\left(1 \pm \Lambda_{+-}{ }^{*} \Lambda_{+-}\right)^{\mp 1 / 4} \exp \left(\frac{1}{2} \Lambda_{+-} c^{*} c^{*}\right) \Omega, \\
\mathscr{U} \prod_{i=1}^{n} c^{(*)}\left(f_{i}\right) \Omega=\prod_{i=1}^{n} c^{(*)}\left(f_{i}\right) \mathscr{U} \Omega,
\end{gathered}
$$

and extend $\mathscr{U}$ by linearity to $D$. The r.h.s. of (5.4) is well defined since $\mathscr{U} \Omega \in D_{\infty}$. Moreover, $\mathscr{U}$ is well defined on $D$ : if $\sum_{l=1}^{N} \alpha_{l} \prod_{i_{l}=1}^{n_{l}} c^{(*)}\left(f_{i_{l}}\right) \Omega=0$, then also $\sum_{l=1}^{N}$ $\alpha_{l} \prod_{i_{l}=1}^{n_{l}} c^{\prime(*)}\left(f_{i}\right) \mathscr{U} \Omega=0$ by virtue of the fact that the $c^{\prime(*)}$ satisfy the CAR (CCR) on $D_{\infty}$ and the lemma. Similarly, using also (4.36) resp. (4.45), it follows that $\mathscr{U}$ is a densely defined isometric operator, and so $\mathscr{U}$ extends to an isometric operator on $\mathscr{F}_{\epsilon}$.

We claim $\overline{\mathscr{U} D}=\mathscr{F}_{\varepsilon}$, i.e., that $\mathscr{U}$ is unitary. Indeed, on $D$ we have for any $v \in \mathscr{H}$

$$
\begin{aligned}
\mathscr{U} \psi(v) & =\mathscr{U}\left[c\left(P_{+} v\right)+c^{*}\left(C_{-} P_{-} v\right)\right]=\left[c^{\prime}\left(P_{+} v\right)+c^{*}\left(C P_{-} v\right)\right] \mathscr{U} \\
& =\psi 4 k(U v) \mathscr{U}, \quad \text { (fermions). }
\end{aligned}
$$


Therefore all $\psi(U v)$ map $\mathscr{U} D$ into itself. Since the $\psi(v)$ are irreducible and $U$ maps $\mathscr{H}$ onto $\mathscr{H}$ it follows that $\overline{\mathscr{U} D}=\mathscr{F}_{a}$. This argument is not valid for bosons since there exist nondense subspaces of $\mathscr{F}_{s}$, mapped into themselves by all $\psi(v)$. However, we have on $D$ for any $v \in \mathscr{H}_{\circ}$

$$
\mathscr{U} \sum_{n=0}^{N}(n !)^{-1} i^{n} \psi(v)^{n}=\sum_{n=0}^{N}(n !)^{-1} i^{n} \psi(q U q v)^{n} \mathscr{M} \quad \text { (bosons) }
$$

Since vectors from $D$ are "entire" analytic vectors for $\psi(v)[5,1 . c$.$] the (strong) limit$ $N \rightarrow \infty$ of the 1.h.s. exists and equals $\mathscr{U} \exp [i \psi(v)]$. Consequently, the limit of the r.h.s. exists, and so it must equal $\exp [i \psi(q U q v)] \mathscr{U}$, as can be seen by using a spectral representation for $\psi(q U q v)$. Thus we have on $D$ for any $v \in \mathscr{H}_{c}$

$$
\mathscr{H} \exp [i \psi(v)]=\exp [i \psi(q U q v)] \mathscr{H} .
$$

Therefore all $\exp [i \psi(q U q v)]$ map $\mathscr{U D}$ into $\overline{\mathscr{U} D}$. Using irreducibility we conclude that $\overrightarrow{\mathscr{U D}}=\mathscr{F}_{s}$, as asserted.

As noted in Section 3, (5.7) implies that $\mathscr{U}$ maps $\tilde{D}$ onto $\tilde{D}$. We now collect these results:

THEOREM 5.2. If $U$ satisfies the assumptions mentioned above, the Bogoliubov transformation generated by $U$ is unitarily implementable. The implementing operator maps $D$ into $D_{\infty}$ and $\tilde{D}$ onto $\tilde{D}$.

We proceed to consider the normal form of $\mathscr{U}$. Let $P, Q$, and $R$ be bounded operators. on $\mathscr{H}_{+}$, then the Wick monomial

$$
\begin{aligned}
& P^{j} Q^{k} R^{l} c^{* j+k} c^{k+2 l+j} \equiv \int \prod_{\rho=1}^{j} d k_{p} d k_{\rho}^{\prime} P\left(k_{p}, k_{p}^{\prime}\right) \prod_{\sigma=1}^{k} d p_{\sigma} d p_{\sigma}^{\prime} Q\left(p_{\sigma}, p_{\sigma}^{\prime}\right) \\
& \quad \times \prod_{\tau=1}^{l} d q_{\tau} d q_{\tau}^{\prime} R\left(q_{\tau}, q_{\tau}^{\prime}\right) c^{*}\left(k_{1}\right) \cdots c^{*}\left(k_{j}\right) c^{*}\left(p_{1}\right) \cdots c^{*}\left(p_{k}\right) c\left(p_{k}^{\prime}\right) \cdots \\
& \times c\left(p_{1}^{\prime}\right) c\left(q_{1}\right) \cdots c\left(q_{l}\right) c\left(q_{l}^{\prime}\right) \cdots c\left(q_{1}^{\prime}\right) c\left(k_{j}^{\prime}\right) \cdots c\left(k_{1}^{\prime}\right)
\end{aligned}
$$

(where, e.g., $P\left(k, k^{\prime}\right)$ is the tempered distribution corresponding to $P$ by the nuclear theorem) has a well-defined meaning as an operator mapping $D$ into $D$ (cf. [1]). Denoting it by $O_{j, k, l}$ one easily verifies that on $D$

$$
\begin{aligned}
O_{j, k, l} c^{*}(f)= & c^{*}(f) O_{j, k, l}+j c^{*}(P f) O_{j-1, k, l} \\
& +k c^{*}(Q f) O_{j, k-1, l}+l O_{j, k, l-1} c\left(\overline{\left[R \mp R^{T}\right] f}\right), \\
c(f) O_{j, k, l}= & O_{j, k, l} c(f)+j O_{j-1, k, l} c\left(P^{*} f\right)+k O_{j, k-1, l} c\left(Q^{*} f\right) .
\end{aligned}
$$


We now introduce an operator

$$
\tilde{\Gamma}(U) \equiv \operatorname{det}\left(1 \pm \Lambda_{+-}{ }^{*} \Lambda_{+-}\right)^{\mp 1 / 4} \sum_{L=0}^{\infty} \mathscr{U}_{L}
$$

with definition domain

$$
D(\hat{\Gamma}(U))=\left\{\phi \in D \mid s \cdot \lim _{N \rightarrow \infty} \sum_{L=0}^{N} \mathscr{U}_{L} \phi \text { exists }\right\}
$$

Here, $\mathscr{U}_{L}$ is the following operator on $D$ :

$$
\mathscr{U}_{L}=2^{-L} \sum_{\substack{i, j, k, l=0 \\ i+j+k+l=L}}^{L}[i ! j ! k ! l !]^{-1} \Lambda_{+-}^{i} \Lambda_{++}^{j}\left(\mp \Lambda_{--}^{T}\right)^{k} \Lambda_{-+}^{l} c^{* 2 i+j+k} c^{k+2 l+j}
$$

By the above and the fact that $\Lambda_{+-}$is H.S. $\mathscr{U}_{L}$ is a well-defined operator, mapping $D$ into $D_{f}$. Clearly, we can write

$$
\hat{\Gamma}(U)=\operatorname{det}\left(1 \pm \Lambda_{+-}^{*} \Lambda_{+-}\right)^{\mp 1 / 4}: \exp \left(\frac{1}{2}\left[\Lambda_{+-} c^{*} c^{*}+\Lambda_{++} c^{*} c+\Lambda_{--} c c^{*}+\Lambda_{-+} c c\right]\right):
$$

We are now in the position to state and prove our main result.

THEOREM 5.3. The domain of $\hat{\Gamma}(U)$ equals $D$. The operator $\mathscr{U}$ is equal to $\hat{\Gamma}(U)$ on $D$ :

$\mathscr{U} \phi=\operatorname{det}\left(1 \pm \Lambda_{+-}{ }^{*} \Lambda_{+-}\right)^{\mp 1 / 4}: \exp \left(\frac{1}{2}\left[\Lambda_{+-} c^{*} c^{*}+\Lambda_{++} c^{*} c+\Lambda_{--} c c^{*}+\Lambda_{-+} c c\right]\right): \phi$,

$\forall \phi \in D$.

Proof. Let $\phi=\prod_{i=1}^{n} c^{*}\left(f_{i}\right) \Omega$, then a term in $\mathscr{U}_{L}$ acting on $\phi$ only gives a nonzero result if $k+2 l \cdot j \leqslant n$. Thus we have for any $N$

$$
\sum_{L=0}^{N} \mathscr{U}_{L} \phi=\sum_{j, k, l=0}^{n} \sum_{i=0}^{M(j . k, l, N)}\left[2^{i} i !\right]^{-1} \Lambda_{+-}^{i} c^{* 2 i} \phi_{j, k, l},
$$

where the notation will be clear. Since $\phi_{j . k . l} \in D$ and $M \rightarrow \infty$ if $N \rightarrow \infty$, Lemma 4.3 shows that $\phi \in D(\hat{\Gamma}(U))$ and that $\hat{\Gamma}(U) \phi \in D_{\infty}$. As a result $D(\hat{\Gamma}(U))=D, \hat{\Gamma}(U)$ $D \subset D_{\infty}$, and on $D$

$$
f(U)=\operatorname{det} \cdots \exp \left(\frac{1}{2} \Lambda_{+-} c^{*} c^{*}\right): \exp \left(\frac{1}{2}\left[\Lambda_{++} c^{*} c+\Lambda_{--} c c^{*}+\Lambda_{-+} c c\right]\right): .
$$

To prove (5.15) we observe that $\hat{\Gamma}(U) \Omega-\mathscr{U} \Omega$ because of (5.3). Therefore it suffices to show that on $D$

$$
\hat{\Gamma}(U) c^{*}(f)=c^{*}(f) \hat{\Gamma}(U), \quad \forall f \in \mathscr{H}_{+} .
$$


(The r.h.s. makes sense since we already know $\hat{\Gamma}(U)$ maps $D$ into $D_{\infty}$.) But on $D$ we have

$$
\begin{aligned}
& \hat{\Gamma}(U) c^{*}(f)=\left[c^{*}(f)+c^{*}\left(\frac{1}{2} \Lambda_{++} f\right) \mp c^{*}\left(\frac{1}{2} \Lambda_{--}^{T} f\right)\right] \hat{\Gamma}(U)+\hat{\Gamma}(U) c\left(\overline{\frac{1}{2}\left[\Lambda_{-+} \mp \Lambda_{-+}^{T}\right]} \bar{f}\right) \\
& \text { (by (5.17), (5.9), and (4.46)) } \\
& \left.=\left[c^{*}\left(U_{++} f\right)-c^{*}\left(\Lambda_{+-} U_{-+} f\right)\right] \hat{\Gamma}(U)+\hat{\Gamma}(U) c \overline{\left(\Lambda_{-+} f\right.}\right) \text { (by (4.6) and (4.8)) } \\
& \left.=\left[c^{*}\left(U_{++} f\right) \pm c\left(\overline{U_{-+} f}\right)\right] \hat{\Gamma}(U)+\hat{\Gamma}(U) c \overline{\Lambda_{-+} f}\right) \\
& +\operatorname{det} \cdots \exp \left(\frac{1}{2} \Lambda_{+-} c^{*} c^{*}\right)\left[\mp c\left(\overline{U_{-+} f}\right)\right]: \exp \cdots: \quad \text { (by (5.17) and (4.47)) } \\
& =c^{\prime *}(f) \hat{\Gamma}(U)+\hat{\Gamma}(U) c \overline{\left(\left[\Lambda_{-+} \mp U_{-+}+\frac{1}{2}\left(\Lambda_{--} \mp \overline{\left.\Lambda_{++}^{T}\right)} \overline{\left.U_{-+}\right] f}\right)\right.\right.} \\
& \text { (by (3.28) and (5.10)) } \\
& =c^{*}(f) \hat{\Gamma}(U) \quad(\text { by }(4.6) \text { and }(4.8)) .
\end{aligned}
$$

The theorem is proven.

It is a well-known property of the creation and annihilation operators that

$$
\Gamma(A) c^{(*)}(f) \Gamma(A)^{*}=c^{(*)}(A f), \quad \forall f \in \mathscr{H}_{+}
$$

if $A$ is a unitary operator on $\mathscr{H}_{+}$. (For a definition of the $\Gamma$-operation and a proof of (5.20) see, e.g., $(5$, l.c.).) Setting

$$
\bar{U}_{--} \equiv U_{++} \equiv A, \quad U_{+-} \equiv U_{-+} \equiv 0,
$$

the operator $\mathscr{U}$ implementing the transformation generated by $U$ also satisfies

$$
\mathscr{U} c^{(*)}(f) \mathscr{U}^{*}=c^{(*)}(A f), \quad \forall f \in \mathscr{H}_{+} .
$$

Since $\mathscr{U}$ is uniquely determined up to a phase factor, and $\mathscr{U} \Omega=\Gamma(A) \Omega=\Omega$, we have $\mathscr{U}=\Gamma(A)$. Thus the theorem gives an explicit expression for $\Gamma(A)$ acting on $D$ :

COROLlaRY 5.4. Let $A$ be a unitary operator on $\mathscr{H}_{+}$. Then one has

$$
\Gamma(A) \phi=: \exp \left([A-1] c^{*} c\right): \phi, \quad \forall \phi \in D .
$$

Proof. This follows from the above and (4.3).

Of course, this corollary can also be easily proven directly.

\section{The General fermion Case}

In this section we obtain the analogs of the results in Section 5 in the case that (4.1) does not hold true. It follows from the unitarity relations (3.26) and the fact that $U_{-+}$is H.S. that $U_{--}$, considcred as an opcrator from $\left(\operatorname{Ker} U_{--}\right)^{\perp}$ to $\left(\operatorname{Ker} U_{--}^{*}\right)^{\perp}$, has 
a bounded inverse, mapping $\left(\operatorname{Ker} U_{--}^{*}\right)^{\perp}$ onto $\left(\operatorname{Ker} U_{--}\right)^{\perp}$. (Here, as in the sequel, the orthocomplement is w.r.t. $\mathscr{H}_{\epsilon}$.) We extend this inverse to $\mathscr{H}_{-}$by setting it equal to zero on $\operatorname{Ker} U_{-}^{*}$ and denote the resulting operator on $\mathscr{H}_{-}$by $U_{-}^{-1}$. In a similar way we define the bounded operator $U_{++}^{*-1}$. If we now again define the associate of $U$ by (4.2) it is not hard to verify that all relations and results in Section 4 again hold true except (4.4). Indeed, (4.4) implies that $U_{--}$, considered as an operator from $\mathscr{H}_{-}$to $\mathscr{H}_{-}$, has an inverse, viz., $1_{--}-\Lambda_{--}$.

Let $\left\{f_{i}\right\}_{i=1}^{L}$ be an o.n. base for $\operatorname{Ker} U_{++}^{*}$. Note that $L<\infty$ since $U_{+-}$is H.S. and that

$$
\left\{f_{i}^{\prime}\right\}_{i=1}^{L} \equiv\left\{C U^{*} f_{i}\right\}_{i=1}^{L}
$$

is an o.n. base for $\operatorname{Ker} U_{++}$. We are now prepared for the following lemma, which shows that the $c^{\prime}(f)$ have a vacuum (cf. also [16, 17]).

LEMMA 6.1. For any $f \in \mathscr{H}_{+}$one has

$$
c^{\prime}(f) \prod_{i=1}^{L} c^{*}\left(f_{i}\right) \exp \left(\frac{1}{2} \Lambda_{+-} c^{*} c^{*}\right) \Omega=0 .
$$

Proof. Assume first $f \in \operatorname{Ker} U_{++}$. Then $c^{\prime}(f)=c^{*}\left(C U_{-+} f\right) \equiv c^{*}(g)$ with $g \in$ Ker $U_{++}^{*}$. Thus for such $f(6.2)$ holds. (Recall that for any $f \in \mathscr{H}_{+}$

$$
c^{*}(f) c^{*}(f)=0, \quad \text { (fermions) }
$$

by virtue of the CAR.) If $f \in\left(\operatorname{Ker} U_{++}\right)^{\perp}$ then $c\left(U_{++} f\right)$ anticommutes with $c^{*}\left(f_{i}\right)$. Using (5.2) we obtain (6.2).

We define

$$
\begin{gathered}
\mathscr{U} \Omega=\operatorname{det}\left(1+\Lambda_{+-}^{*} \Lambda_{+-}\right)^{-1 / 4} \prod_{i=1}^{L} c^{*}\left(f_{i}\right) \exp \left(\frac{1}{2} \Lambda_{+-} c^{*} c^{*}\right) \Omega, \\
\mathscr{U} \prod_{i=1}^{n} c^{(*)}\left(e_{i}\right) \Omega=\prod_{i=1}^{n} c^{(*)}\left(e_{i}\right) \mathscr{U} \Omega
\end{gathered}
$$

and extend $\mathscr{U}$ by linearity to $D$. (Here, as in the sequel, the products are in the natural order of the indices.) Since $A_{+-} C f_{i}=0, i=1, \ldots, L$, it follows from (4.47) that $c\left(f_{i}\right)$ commutes with $\exp (\cdots)$. Therefore $\|\mathscr{U} \Omega\|=1$. In the same fashion as in Section 5 we now obtain:

THEOREM 6.2. If $U$ satisfies the assumptions mentioned above, the Bogoliubov transformation generated by $U$ is unitarily implementable. The implementing operator maps $D$ into $D_{\infty}$.

We again define $\hat{\Gamma}(U)$ by (5.11)-(5.13). By inspection of the proof of Theorem 5.3 we conclude that also in this case $f(U)$ maps $D$ into $D_{\infty}$ and satisfies on $D$

$$
\hat{f}(U) c^{*}(f)=c^{* *}(f) \hat{\Gamma}(U), \quad \forall f \in \mathscr{H}_{+} .
$$


Moreover one concludes from the second line of (5.19) by using (4.3) that

$$
\hat{\Gamma}(U) c^{*}(f)=0, \quad \forall f \in \operatorname{Ker} U_{++}
$$

and so by (6.6) that

$$
c(f) \hat{\Gamma}(U)=0, \quad \forall f \in \operatorname{Ker} U_{++}^{*}
$$

We also have occasion to use the operator $\hat{\Gamma}(-U)$. Clearly it satisfies (6.7) and (6.8), and

$$
\hat{\Gamma}(-U) c^{*}(f)=-c^{*}(f) \hat{\Gamma}(-U), \quad \forall f \in \mathscr{H}_{+} .
$$

Let now $P$ be the set of all partitions of the index set $\{1, \ldots, L\}$ into two subsets, i.e., an element $\rho \in P$ is given by two disjoint subsets $\left\{\rho_{1}, \ldots, \rho_{l}\right\}$ and $\left\{\rho_{l+1}, \ldots, \rho_{L}\right\}$ (the indices are by convention in the natural order). We define a function on $P$ by

$$
\operatorname{sgn} \rho=\operatorname{sgn}\left(\rho_{l+1}, \ldots, \rho_{L}, \rho_{1}, \ldots, \rho_{l}\right),
$$

i.e., sgn $\rho$ is the sign of the permutation of the indices $\{1, \ldots, L\}$ occurring on the r.h.s. We are now ready for the main result of this section.

THEOREM 6.3. The operator $\mathscr{U}$ satisfies

$$
\mathscr{U} \phi=\sum_{\rho \in P} \operatorname{sgn} \rho \prod_{i=1}^{l} c^{*}\left(f_{p_{i}}\right) \hat{\Gamma}\left((-)^{L} U\right) \prod_{i=l+1}^{L} c\left(f_{\rho_{i}}^{\prime}\right) \phi, \quad \forall \phi \in D .
$$

Proof. Since (6.11) holds if $\phi=\Omega$ (cf. (6.4)) it is sufficient to prove

$$
\mathscr{U}^{\prime} c^{*}(f)=c^{*}(f) \mathscr{U}^{\prime}, \quad \forall f \in \mathscr{H}_{+}
$$

where $\mathscr{U}^{\prime}$ denotes the operator on the r.h.s. of (6.11). First assume $f \in\left(\operatorname{Ker} U_{++}\right)^{\perp}$. Then $c^{*}(f)$ anticommutes with $c\left(f_{i}^{\prime}\right)$. Using now (6.6) ( $L$ even) or (6.9) ( $L$ odd) and the fact that $c^{*}(f)$ anticommutes with $c^{*}\left(f_{i}\right)$ we obtain (6.12). We are therefore done if we can show

$$
\mathscr{U}^{\prime} c^{*}\left(f_{i}^{\prime}\right)=c\left(f_{i}\right) \mathscr{U}^{\prime}, \quad i=1, \ldots, L
$$

Now from the CAR and (6.7) and (6.8) it is clear that, neglecting signs, the same operators occur on the r.h.s. and 1.h.s. of (6.13). However, it is not difficult to verify that our definition of sgn $\rho$ is just such as to guarantee that they have the same signs. The theorem is proven.

\section{Comparison With [1]. Connection between CAR- AND CCR-Transformations}

As noted before, our approach and notation are inspired by the Bogoliubov transformations that arise in theories of relativistic particles in external fields. The general case treated in this paper corresponds to the case of neutral particles. For the 
quantization of the classical (i.e., single-particle) theory, which is formulated in the Hilbert space $\mathscr{H}$, one then only uses the "positive-energy subspace" $\mathscr{H}_{+}$, i.e., one only considers $\mathscr{F}_{\epsilon}\left(\mathscr{H}_{+}\right)$. In contrast, for the case of charged particles one uses $\mathscr{F}_{\epsilon}(\mathscr{H})$, which is canonically isomorphic to $\mathscr{F}_{e}\left(\mathscr{H}_{+}\right) \otimes \mathscr{F}_{e}\left(\mathscr{H}_{+}\right)$. The first factor, $\mathscr{F}_{e}\left(\mathscr{H}_{+}\right)$, is then associated with the particles, while the second factor is associated with the antiparticles (cf. also [18]). The Bogoliubov transformations occurring in the latter case have been considered in detail in [1]. In this section we want to derive the main results of [1], viz., Theorems 4.1 and 5.1, from the general case considered here. Moreover, we want to point out a connection between the fermion and boson transformations considered in [1] and to establish its analog in the general case.

The structure of this special case is such that the analogs of the general results obtained in this paper could be derived in a quite similar fashion. However, in [1] we did not introduce a copy of the Hilbert space on which Fock space is built. In fact, for such transformations this procedure is an auxiliary device that is not only unnecessary, but even quite unnatural. This circumstance and the situation sketched above forces us to cause the reader some inconvenience. We ask that he mentally replace in Sections 2-6 every index + and - by 1 resp. 2 , and the symbol $A$, where $A=\mathscr{H}$, $C, q, U$, or $A$ by $\hat{A}$. (Thus, e.g., (3.2) now reads $\hat{q}=P_{1}-P_{2}$, the first relation of (4.2) $\hat{A}_{22}= \pm\left(1_{22}-\hat{U}_{22}{ }^{-1}\right)$, and we now have $\hat{\mathscr{H}}=\hat{\mathscr{H}}_{1} \oplus \hat{\mathscr{H}}_{2}$.) However, the old notation will be used for new spaces and operators to be introduced below.

We can now define the Bogoliubov transformations studied in [1] as the transformations generated by those (pseudo-) unitary operators $\hat{\theta}$ on $\mathscr{H}$, commuting with $\hat{C}$, that have an additional property: $\hat{\mathscr{H}}_{1}$ can be decomposed as the direct sum of two subspaces $\mathscr{H}_{+}$and $\mathscr{H}_{-}$(with corresponding projections $P_{+}$and $P_{-}$) such that $\hat{U}_{11}$ maps $\mathscr{H}_{\epsilon}$ into $\mathscr{H}_{\epsilon}$ and $\hat{U}_{21}$ (considered in the natural way as an operator on $\hat{\mathscr{H}}_{1}$ ) maps $\mathscr{H}_{\epsilon}$ into $\mathscr{H}_{-\epsilon}$. We assume from now on that $\hat{U}$ is such an operator. For notational convenience and because this corresponds to applications we further assume just as in [1] that

$$
\mathscr{H} \equiv \hat{\mathscr{H}}_{1}=L^{2}\left(R^{m}, d p\right)^{2 M}, \quad \mathscr{H}_{+}, \mathscr{H}_{-}=L^{2}\left(R^{m}, d p\right)^{M} .
$$

We can then define an operator $U$ on $\mathscr{H}$ by setting

$$
\left(\begin{array}{cc}
U_{++} & 0 \\
0 & \overline{U_{--}}
\end{array}\right) \equiv \hat{U}_{11} ; \quad\left(\begin{array}{cc}
0 & \overline{U_{+-}} \\
U_{-+} & 0
\end{array}\right) \equiv \hat{U}_{21} .
$$

It is straightforward to verify that $U$ is a unitary operator resp. pseudo-unitary operator w.r.t. $q \equiv P_{+}-P_{-}$. Conversely, if an operator $U$ on $\mathscr{H}$ has this property, and we define $\hat{U}_{11}$ and $\hat{U}_{21}$ by (7.2), and set

$$
\hat{U}_{22} \equiv \overline{\hat{U}_{11}}, \quad \hat{U}_{12} \equiv \overline{\hat{U}_{21}},
$$

then $U$ is a unitary operator resp. pseudo-unitary operator w.r.t. $\hat{q}=P_{1}-P_{2}$ on $\mathscr{H}$, commuting with $\hat{C}$. If we now introduce particle and antiparticle creation and annihilation operators by setting

$$
c^{(*)}(v) \equiv a^{(*)}\left(P_{+} v\right)+b^{(*)}\left(P_{-} v\right), \quad \forall v \in \mathscr{H},
$$


then we can write the transformation generated by the inverse of $\hat{U}$, viz.,

$$
c^{\prime}(v)=c\left(\hat{U}_{11} v\right) \pm c^{*} \overline{\left(\hat{U}_{21} v\right)}, \quad \forall v \in \mathscr{H}
$$

(cf. (3.28)) as

$$
\begin{array}{ll}
a^{\prime}(f)=a\left(U_{++} f\right) \pm b^{*}\left(\overline{U_{-+} f}\right), & \forall f \in \mathscr{H}_{+} \\
b^{\prime}(\bar{g})=b\left(\overline{U_{--} g}\right) \pm a^{*}\left(U_{+-} g\right), & \forall g \in \mathscr{H}_{-} .
\end{array}
$$

This is just Eq. (2.25) in [1].

From now on we assume that the transformation is unitarily implementable, i.e., that $\hat{U}_{21}$ is H.S. Note that this is equivalent to $U_{t-}$ and $U_{-+}$being H.S. In addition, we assume first (in the fermion case) that $\operatorname{Ker} \hat{U}_{11}=0$ or, equivalently, that $\operatorname{Ker} U_{++}=$ $\operatorname{Ker} U_{--}=0$. Then we can define a bounded operator $\Lambda$ on $\mathscr{H}$ by one of the equivalent relations (4.2)-(4.5). Note that $\Lambda$ satisfies (4.6) if and only if $U$ satisfies (3.24), but that (3.24) does not need to hold true. It is straightforward to verify that the associates of $\hat{U}$ and $U$ are related by

$$
\begin{aligned}
& \hat{\Lambda}_{\mathrm{11}}=\mp \hat{\Lambda}_{22}^{T}=\left(\begin{array}{cc}
\Lambda_{++} & 0 \\
0 & \mp \Lambda_{--}^{T}
\end{array}\right), \\
& \hat{\Lambda}_{12}=\left(\begin{array}{cc}
0 & \Lambda_{+-} \\
\mp \Lambda_{+-}{ }^{T} & 0
\end{array}\right), \\
& \hat{\Lambda}_{21}=\left(\begin{array}{cc}
0 & \mp \Lambda_{-+}^{T} \\
\Lambda_{-+} & 0
\end{array}\right) .
\end{aligned}
$$

Moreover one has

$$
\operatorname{det}\left(1 \pm \hat{\Lambda}_{12}^{*} \hat{\Lambda}_{12}\right)^{\mp 1 / 4}=\operatorname{det}\left(1 \pm \Lambda_{+-}^{*} \Lambda_{+--}\right)^{\mp 1 / 2}
$$

Indeed, by (7.7),

$$
\hat{\Lambda}_{12} * \hat{\Lambda}_{12}=\left(\begin{array}{cc}
\overline{\Lambda_{+-} \Lambda_{+-}^{*}} & 0 \\
0 & \Lambda_{+-}^{*} \Lambda_{+-}
\end{array}\right)
$$

The assertion therefore follows from the fact that $A A^{*}$ has the same eigenvalues (including multiplicity) as $A^{*} A$ if $A$ is compact. From (7.7) and (7.8) it easily follows that (the substitute of) Eq. (5.15) can be written as

$$
\begin{array}{r}
\mathscr{T} \phi=\operatorname{det}\left(1 \pm \Lambda_{+-}{ }^{*} \Lambda_{+-}\right)^{\mp 1 / 2}: \exp \left(\Lambda_{+-} a^{*} b^{*}+\Lambda_{++} a^{*} a+\Lambda_{--} b b^{*}+\Lambda_{-+} b a\right): \phi, \\
\forall \phi \in D .
\end{array}
$$

This equation (and, of course, its interpretation) is the content of Theorem 4.1 in [1]. The notation $\tilde{\Gamma}(U)$, used there, denotes the same operator that is denoted by $\hat{\Gamma}(\hat{U})$ here. 
Let us now consider the general fermion case, i.e., $\operatorname{Ker} \hat{\theta}_{11} \neq 0$ or, equivalently, $\operatorname{Ker} U_{++} \neq 0$ or $\operatorname{Ker} U_{--} \neq 0$ or both. One can then still define the associate of $U$ by (4.2) or (4.3) if one interprets $U_{--}^{-1}$ resp. $U_{++}^{*-1}$ as described in Section 6, and again one verifies that (7.7) and (7.8) hold true. By making a suitable choice for the o.n. base of $\operatorname{Ker} \theta_{11}^{*}$ occurring in (6.1) one can now easily derive Theorem 5.1 in [1] from Theorem 6.3.

Our last topic is the mentioned connection between fermion and boson transformations. This connection rests on the observation that there is a one-one relation between pseudo-unitary operators and unitary operators such that $U_{-}$has a bounded inverse. More generally, this relation can be defined for all bounded operators $U$ on $\mathscr{H}$ such that $U_{--}$has a bounded inverse. Indeed, the transformation $\phi: U \rightarrow Z$ defined by

$$
\begin{array}{ll}
Z_{--}=U_{--}^{-1}, & Z_{+-}=U_{+-} U_{--}^{-1}, \\
Z_{-+}=-U_{--}^{-1} U_{-+}, & Z_{++}=U_{++}-U_{+-} U_{--}^{-1} U_{-+}
\end{array}
$$

is a bijection of the set of such operators with inverse $\phi$. It is straightforward to verify that $U$ is unitary/pseudo-unitary if and only if $Z$ is pseudo-unitary/unitary. Let us assume that, e.g., $U$ is unitary and $Z$ pseudo-unitary. By comparing (7.11) and (4.2) we see that

$$
Z=1+q \Lambda
$$

Similarly, we have

$$
U=1+\Lambda^{\prime}
$$

where $\Lambda^{\prime}$ is the associate of $Z$. (Note also that (7.11) is equivalent to

$$
U P_{+}-P_{+} Z+U P_{-} Z=Z P_{+}-P_{+} U+Z P_{-} U=P_{-}
$$

and compare this and (7.12) and (7.13) with (4.4) and (4.5).)

We now make the further assumption that the CAR-transformation generated by $U$ is unitarily implementable in $\mathscr{F}_{a}(\mathscr{H})$. Because of $(7.11)$ this is the case if and only if the CCR-transformation generated by $Z$ is unitarily implementable in $\mathscr{F}_{s}(\mathscr{H})$. By virtue of $(7.10)$ we then have in $\mathscr{F}_{a}(\mathscr{H})$ on $D$

$$
\mathscr{U}=\operatorname{det}\left(1+\Lambda_{+-}^{*} \Lambda_{+-}\right)^{-1 / 2}: \exp \left(\Lambda_{+-} a^{*} b^{*}+\Lambda_{++} a^{*} a+\Lambda_{--} b b^{*}+\Lambda_{-+} b a\right)
$$

while we have in $\mathscr{F}_{s}(\mathscr{H})$ on $D$

$$
\mathscr{Z}=\operatorname{det}\left(1-\Lambda_{+-}^{\prime} \Lambda_{+-}^{\prime}\right)^{1 / 2}: \exp \left(\Lambda_{+-}^{\prime} a^{*} b^{*}+\Lambda_{++}^{\prime} a^{*} a+\Lambda_{--}^{\prime} b b^{*}+\Lambda_{-+}^{\prime} b a\right):
$$

We claim that

$$
(\Omega, \mathscr{U} \Omega)=(\Omega, \mathscr{Z} \Omega)
$$


where on the 1.h.s. (r.h.s.) $\Omega$ is the $\mathscr{F}_{a}(\mathscr{H})-\left(\mathscr{F}_{s}(\mathscr{H})-\right)$ vacuum. Clearly, (7.17) holds if and only if

$$
\operatorname{det}\left(1+\Lambda_{+-}^{*} \Lambda_{+-}\right) \operatorname{det}\left(1-\Lambda_{+-}^{\prime}{ }^{*} \Lambda_{+-}^{\prime}\right)=1 .
$$

By (7.12) and (7.13) and (pseudo-) unitarity this is equivalent to

$$
\operatorname{det}\left(Z_{-\_}^{*} Z_{-\_}\right) \operatorname{det}\left(U_{-\_}^{*} U_{--}\right)=1 \text {. }
$$

Now $U_{--}^{*} U_{--}$has the same eigenvalues as $U_{--} U_{--}^{*}$, and therefore (7.19) follows from the relation $Z_{-\_}^{*} Z_{--} U_{--} U_{-}^{*}=1 \ldots$ and a well-known property of infinite determinants [13]. Setting

$$
d \equiv(\Omega, \mathscr{U} \Omega)=(\Omega, \mathscr{Z} \Omega)
$$

we can write (7.15) and (7.16), using (7.12) and (7.13),

$$
\begin{aligned}
& \mathscr{U}=d: \exp \left(Z_{+-} a^{*} b^{*}+\left(Z_{++}-1\right) a^{*} a-\left(Z_{--}-1\right) b b^{*}-Z_{-+} b a\right): \\
& \mathscr{Z}=d: \exp \left(U_{+-} a^{*} b^{*}+\left(U_{++}-1\right) a^{*} a+\left(U_{--}-1\right) b b^{*}+U_{-+} b a\right):
\end{aligned}
$$

where (7.21) holds on $D$ in $\mathscr{F}_{a}(\mathscr{H})$, and (7.22) holds on $D$ in $\mathscr{F}_{s}(\mathscr{H})$.

Summarizing, we have established that there is a one-one relation between unitary operators $U$ such that $U_{--}$has a bounded inverse and pseudo-unitary operators $Z$. This relation is such that the CAR-transformation generated by $U$ is unitarily implementable in $\mathscr{F}_{a}(\mathscr{H})$ if and only if the CCR-transformation generated by $Z$ is unitarily implementable in $\mathscr{F}_{s}(\mathscr{H})$. The implementing operators $\mathscr{U}$ and $\mathscr{Z}$ can be expressed in $Z$ resp. $U$ through (7.21) resp. (7.22), and have the same vacuum expectation value.

In the general case considered in Sections 4 and 5 one can also define $Z$ by (7.11) and then (7.12)-(7.14) again hold true. (Here and from now on the old notation is used again.) Therefore it is natural to ask whether the analogous correspondence persists in the general case. However, from the fact that $U$ commutes with $C$ it follows, using (7.11), that

$$
C Z C=Z^{-1}
$$

Hence, if we require that $Z$ also generate a Bogoliubov transformation, i.e., that it also commute with $C$, we must have $Z=Z^{-1}$ and so by symmetry $U=U^{-1}$. As a result the unitary operator of the pair $U, Z$, e.g., $U$, fulfills

$$
U=Q-R,
$$

where $Q$ and $R$ are mutually orthogonal projections that commute with $C$ and satisfy

$$
Q+R=1 .
$$

Thus the analogous correspondence only exists for a very limited class of operators in the general case. (Note that this class does not contain the special operators $\hat{U}$ con- 
sidered above.) If $U, Z$ is a pair in this class (with, e.g., $U$ unitary) for which the transformations are unitarily implementable one obtains as the analogs of (7.21) and (7.22)

$$
\begin{aligned}
& \mathscr{U}=d: \exp \left(\frac{1}{2}\left[Z_{+-} c^{*} c^{*}+\left(Z_{++}-1\right) c^{*} c-\left(Z_{--}-1\right) c c^{*}-Z_{-+} c c\right]\right) \\
& \mathscr{Z}=d: \exp \left(\frac{1}{2}\left[U_{+-} c^{*} c^{*}+\left(U_{++}-1\right) c^{*} c+\left(U_{--}-1\right) c c^{*}+U_{-+} c c\right]\right):
\end{aligned}
$$

where (7.26) holds on $D$ in $\mathscr{F}_{a}\left(\mathscr{H}_{+}\right)$and (7.27) holds on $D$ in $\mathscr{F}_{s}\left(\mathscr{H}_{+}\right)$, and where

$$
d \equiv \operatorname{det}\left(1+Z_{+-}{ }^{*} Z_{+-}\right)^{-1 / 4}=\operatorname{det}\left(1-U_{+-}{ }^{*} U_{+-}\right)^{1 / 4} .
$$

We finally mention that a pair $U, Z$ as considered above occurs in the treatment of the external field problem (cf. [19, especially Theorem 2.10; 20, especially Remark 4.2]).

\section{REFERENCES}

1. S. N. M. RuiJsenaArs, J. Mathematical Phys. 18 (1977), 517-526.

2. S. N. M. RuIJSENAARs, Neutral particles in external fields (in preparation).

3. F. A. Berezin, "The Method of Second Quantization," Academic Press, New York, 1966.

4. M. ReED AND B. Simon, "Methods of Modern Mathematical Physics I. Functional Analysis," Academic Press, New York, 1972.

5. M. ReEd AND B. Simon, "Methods of Modern Mathematical Physics II. Fourier Analysis, SelfAdjointness," Academic Press, New York, 1975.

6. N. Bogoliubov, J. Phys. USSR 11 (1947), 23-32.

7. K. O. FRIEDRICHS, "Mathematical Aspects of the Quantum Theory of Fields," Interscience, New York, 1953.

8. P. Kristensen, L. Mejlbo, and E. Thue Poulsen, Comm. Math. Phys. 6 (1967), 29-48.

9. I. E. Segal, "Mathematical Problems of Relativistic Physics," American Mathematical Society, Providence, R.I., 1963.

10. G. G. Емсн, "Algebraic Methods in Statistical Mechanics and Quantum Field Theory," Interscience, New York, 1972.

11. D. Shale, Trans. Amer. Math. Soc. 103 (1962), 149-167.

12. D. Shale and W. F. Stinespring, J. Math. Mech. 14 (1965), 315-322.

13. B. Simon, Advances in Math. 24 (1977), 244-273.

14. B. Schroer, R. Seiler, And J. A. Swieca, Phys. Rev. D 2 (1970), 2927-2937.

15. R. SeILer, Comm. Math. Phys. 25 (1972), 127-151.

16. G. Labonté, Comm. Math. Phys. 36 (1974), 59-72.

17. K. Fredenhagen, Comm. Math. Phys. 52 (1977), 255-266.

18. A. S. Wightman, Relativistic Wave Equations as Singular Hypcrbolic Systems, in "Partial Differential Equations" (D. Spencer, Ed.) Symp. Pure Math. 23, pp. 441-477. American Mathematical Society, Providence, R.I., 1973.

19. S. N. M. RujusenaArs, J. Mathematical Phys. 18 (1977), 720-737.

20. S. N. M. RuiJsenaars, Comm. Math. Phys. 52 (1977), 267-294. 